\title{
Trends in Carbon, Oxygen, and Nitrogen Core in the X-ray Absorption Spectroscopy of Carbon Nanomaterials: A Guide for the Perplexed
}

Sami Sainio,* Niklas Wester, Anja Aarva, Charles J. Titus, Dennis Nordlund, Esko I. Kauppinen, Elli Leppänen, Tommi Palomäki, Jessica E. Koehne, Olli Pitkänen, Krisztian Kordas, Maria Kim, Harri Lipsanen, Miran Mozetič, Miguel A. Caro, M. Meyyappan, Jari Koskinen, and Tomi Laurila

Cite This: J. Phys. Chem. C 2021, 125, 973-988

Read Online

ABSTRACT: Successful deployment of carbon nanomaterials in many applications, such as sensing, energy storage, and catalysis, relies on the selection, synthesis, and tailoring of the surface properties. Predictive analysis of the behavior is difficult without detailed knowledge of the differences between various carbon nanomaterials and their surface functionalization, thus leaving the selection process to traditional trial-and-error work. The present characterization fills this knowledge gap for carbon nanomaterial surface properties with respect to chemical states and functionalization. We present an overview of the chemical trends that can be extracted from soft X-

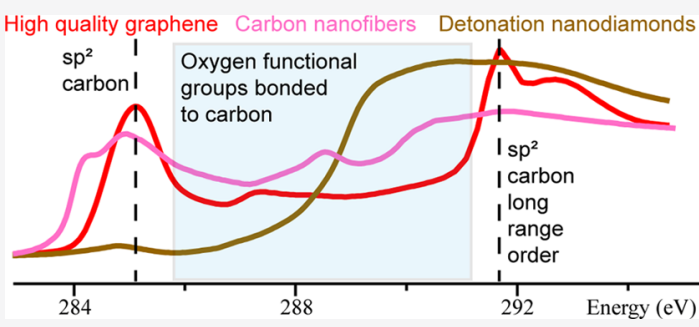
ray absorption spectroscopy (XAS) spectra on an extended set of nonideal carbon nanomaterials as a function of $\mathrm{sp}^{2}$ bonded carbon and bond ordering. In particular, the surface chemical state, the presence of long-range order in the carbon matrix, and a qualitative estimation of the amount of oxygen and nitrogen and their respective functional group formation on the material surface, together with the detailed material fabrication parameters, are reported. The results expand our understanding of carbon nanomaterial functionalization, which can support material selection in practice, provided that the specifications of the application are known.

\section{INTRODUCTION}

Carbon nanomaterials (CNMs) are used in a wide range of applications, including sensors, ${ }^{1-4}$ energy storage and conversion, ${ }^{5,6}$ catalysis, ${ }^{7}$ advanced composites, ${ }^{8}$ drug delivery platforms, ${ }^{9}$ and many more. They are also used in several industrial applications as wear-protective coatings for diskdrive read head coating using a diamond-like carbon, ${ }^{10}$ touch panels with conductive carbon nanotubes $(\mathrm{CNTs})^{11}$ and graphene, ${ }^{5}$ conductive additives in electrode materials for batteries, additives in anticorrosion primers, precursors for thermal dissipation films, ${ }^{12}$ and anode materials in lithium-ion batteries, $^{13}$ to name a few examples. The surface chemistry plays a key role in the sensitivity and selectivity of sensors, ${ }^{14}$ surface wettability, ${ }^{15}$ covalent binding of enzymes and antibodies, ${ }^{16}$ cell viability, ${ }^{17}$ and tribological properties. ${ }^{18}$ To broaden the applicability of these often quite unideal carbon nanomaterials into new industrial-scale applications, it is crucial to develop a mechanistic understanding of their surface chemistry (or a better understanding of the functionality of their surface chemistry). As shown in ref 19 , there is a serious lack of (i) systematic characterization of carbon nanomaterials and (ii) standardized protocols for characterizing the surface functional groups of carbon nanomaterials. Furthermore, most past studies do not explicitly state how the studied materials were grown or prepared, and in some cases, only the vendor information is available. Thus, our ability to tailor carbonaceous nanomaterials for specific applications is limited. Here, we provide details of the fabrication process for a wide range of extensively used carbon nanomaterials, as well as the results of $\mathrm{X}$-ray absorption spectroscopy (XAS) analysis, to be able to provide a consistent and systematically constructed dataset, which allows for more robust extraction of trends. Note that this paper is intended to provide information about the overall trends that can be seen in these often nonideal carbon nanomaterials used in the actual applications. Similar approaches can be found for conjugated polymers, ${ }^{20}$ carbonyl-containing polymers, ${ }^{21}$ asphaltenes, ${ }^{22}$ polycyclic aromatic hydrocarbons, ${ }^{23}$ natural organic carbon, ${ }^{24-26}$ soil organic carbon, ${ }^{27}$ a wide array of molecular carbon compounds in various groupings, ${ }^{28-30}$ to name a few. This paper is not intended to be an in-depth investigation of the detailed surface chemical properties of a single ideal carbon allotrope that would be targeted solely for research purposes. The latter can

Received: September 21, 2020

Revised: December 9, 2020

Published: December 24, 2020 


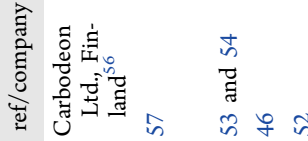
8
:
ज्ञ
के

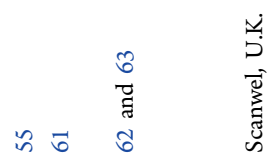

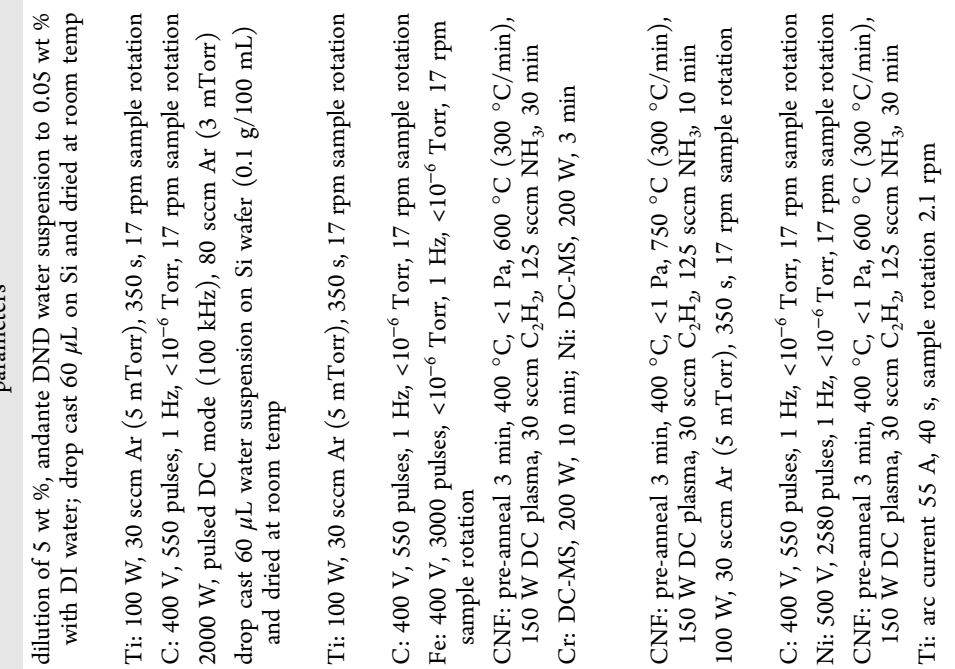

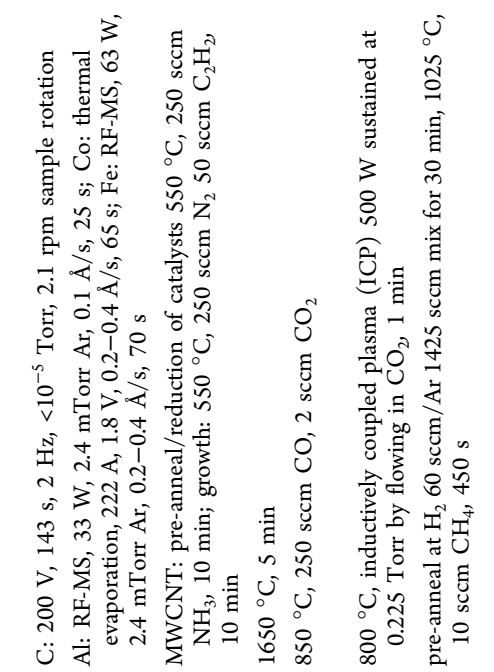

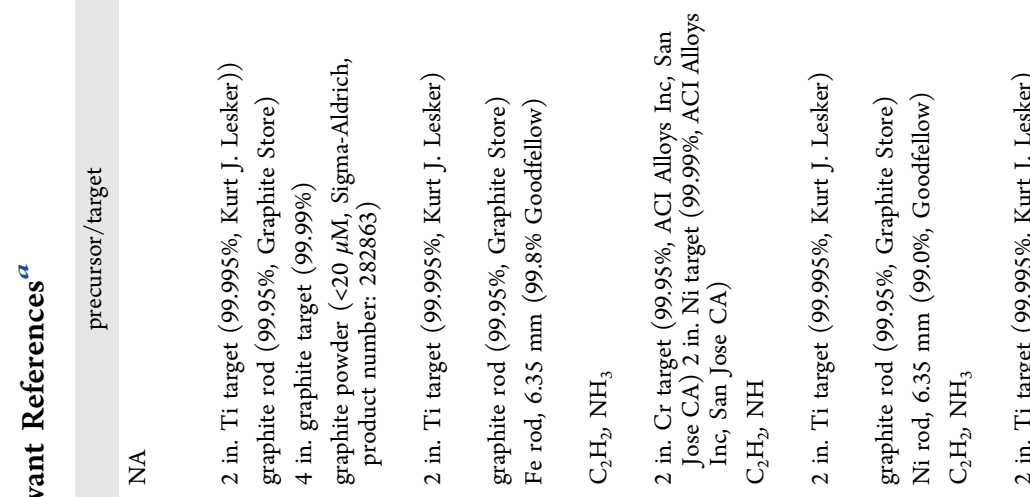

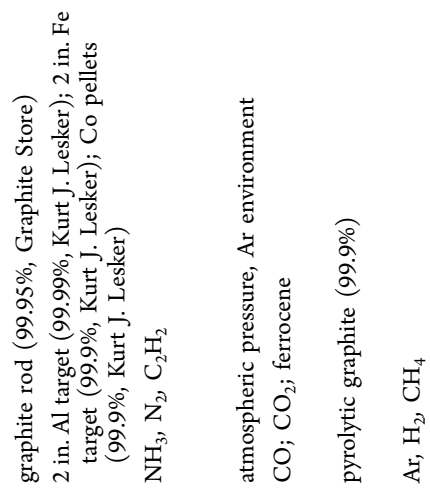

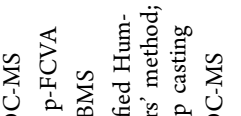
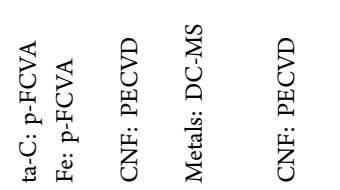

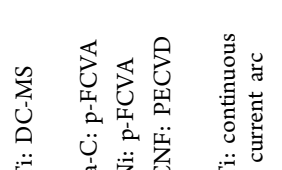

童
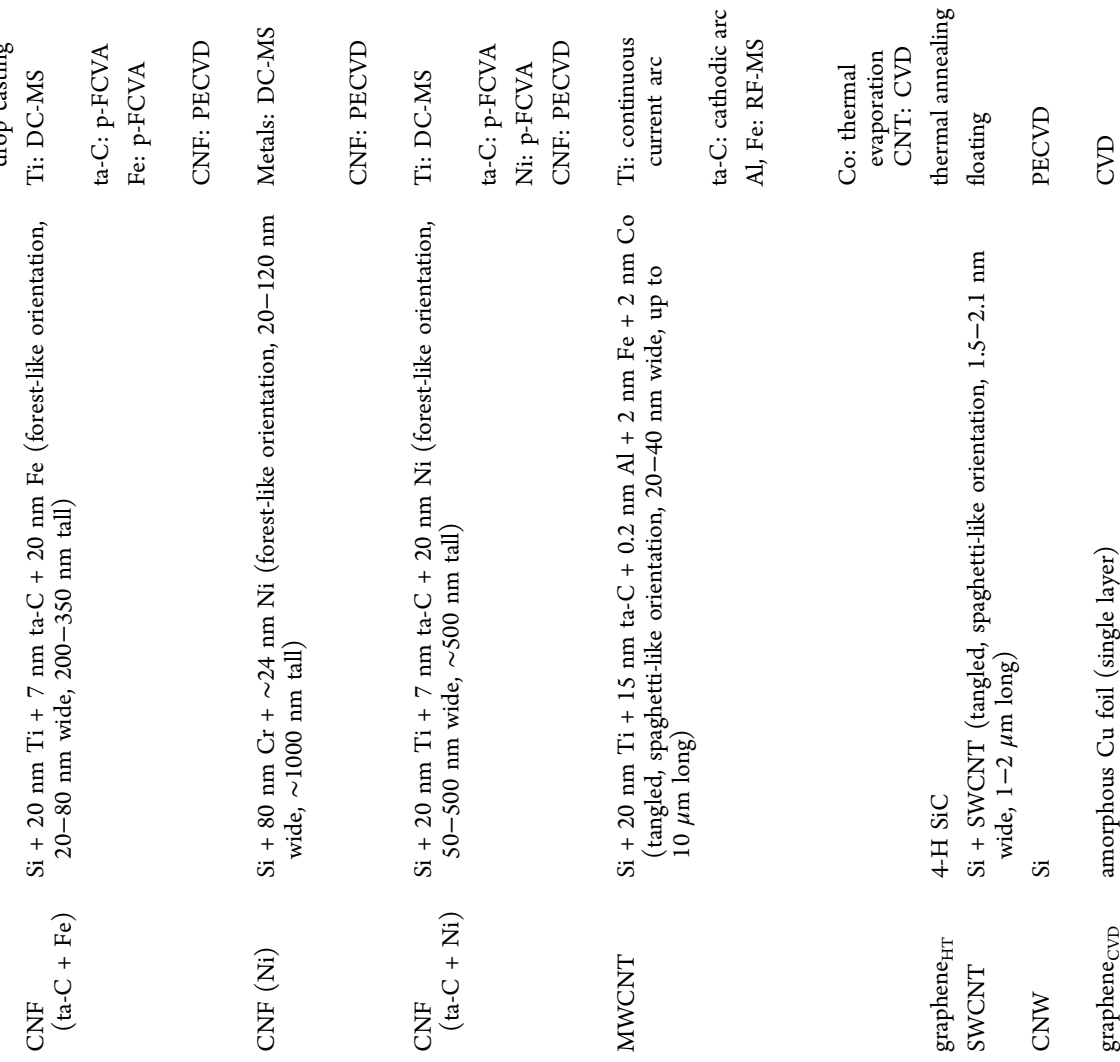

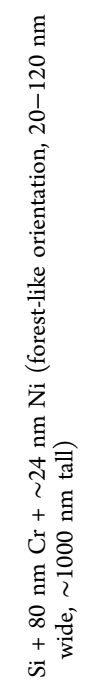

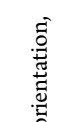

ن

蒫

곤

空泀

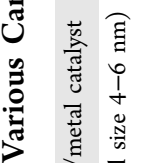

อั

这

思

$\stackrel{+}{+} \quad$ i

青自

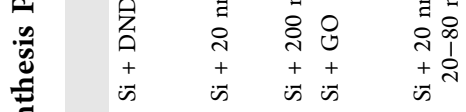

自

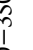

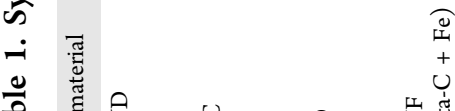

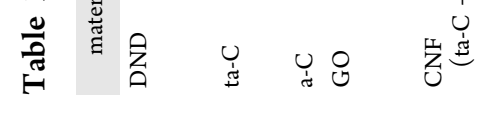

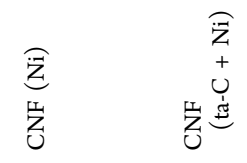

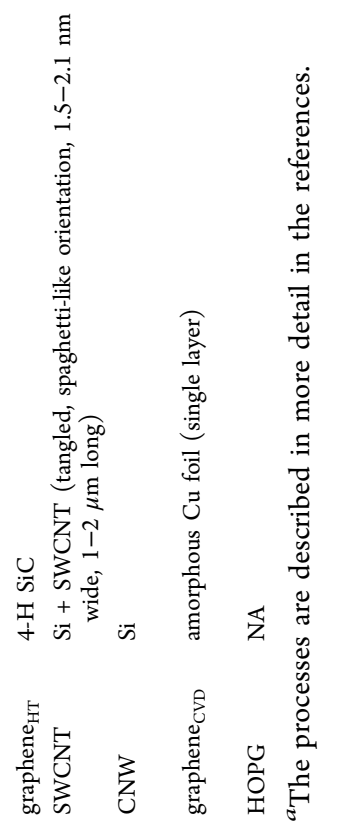


be found in multiple publications, e.g., for graphene, ${ }^{31}$ carbon nanotubes, ${ }^{32-34}$ graphitic carbon nitride $\left(\mathrm{g}-\mathrm{C}_{3} \mathrm{~N}_{4}\right),{ }^{35} \mathrm{C} 60 /$ $\mathrm{C} 70,{ }^{36}$ diamond, ${ }^{37}$ nanodiamonds (NDs), ${ }^{38}$ and amorphous carbon (a-C). ${ }^{39}$ An interesting one-dimensional carbon allotrope carbyne was also proposed for the first time already in $1967^{40}$ and further studied more recently. ${ }^{41,42}$ However, as it is, at the present stage, not utilized extensively in any widespread applications in contrast to the other carbon allotropes as well as due to the lack of appropriate material sample, carbyne is not included in the material portfolio of this study and will not be discussed further.

In the field of electrochemical applications, there is a longstanding debate regarding which properties of carbon nanomaterials are the most critical in determining their electrocatalytic performance. One of the proposed hypotheses claimed that the edge planes are more active than the basal planes in carbon nanomaterials when they are both present. $^{43,44}$ Numerous publications also show possible connections between the amount of the $\mathrm{sp}^{2} / \mathrm{sp}^{3}$ fraction and the surface oxygen content. ${ }^{45,46}$ In addition to the total oxygen content, the types of functional groups present on the surface as well as species dissolved subsurface have been observed to affect the electrocatalytic properties. ${ }^{47}$ Thus, to understand the performance of a given carbon nanomaterial in a specific application, such as those listed above, functional groups and other physicochemical features of the surface must be known accurately. It must be noted that it has also been speculated that the residual catalyst metals may in fact have a dominating effect on the electrochemical properties of carbonaceous nanomaterials, although there also exist arguments against this claim, especially when considering the effects of additives combined with graphene. ${ }^{48}$ While this work focuses on the chemistry of carbon nanomaterials, disregarding the presence of trace metals, we acknowledge that the metal catalysts used in many of the fabrication processes of the carbon nanomaterials are also likely to play a significant role in their performance in various applications. Detailed analysis of the amount of trace elements and their chemical states for the current set of carbon materials will be considered in our future work. Hence, our primary goal here is to provide a chemical and structural map that can be used to navigate through the rich landscape of carbon nanomaterials used in practical applications.

\section{EXPERIMENTAL SECTION}

Material Fabrication. Highly ordered pyrolytic graphite (HOPG) was acquired from Scanwel (see Table 1 for details). To expose a fresh surface, a scotch tape method ${ }^{49}$ was used to peel several layers off from the HOPG prior to measurement. Single-digit detonation nanodiamond (DND) in a solution with a $\zeta$ potential of $+40 \mathrm{mV}$ prepared from detonation soot and chemically purified to remove the $\mathrm{sp}^{2}$ carbon and metal impurities was obtained from Carbodeon. All other materials were deposited specifically for this work. The carbon nanomaterials in water solution were drop cast onto borondoped $\langle 100\rangle$ Si wafers (resistance $<0.005 \Omega \cdot \mathrm{cm}$ ). Graphite oxide (GO) was synthesized from synthetic graphite powder (Sigma-Aldrich) following a modified Hummers' method ${ }^{50,51}$ and exfoliated into GO by sonication as described in ref 52 . The suspension was dried after sonication to obtain GO powder that was further dispersed in deionized (DI) water to achieve a solution with $0.1 \mathrm{~g} / 100 \mathrm{~mL}$. A $60 \mu \mathrm{L}$ drop of this solution was then drop cast on a $\mathrm{Si}$ substrate and dried overnight. A $200 \mathrm{~nm}$ thick a-C layer was deposited by closed- field unbalanced magnetron sputtering (CFUBMS) as described in refs 53 and 54. Seven nanometer thick tetrahedral a-C (ta-C) samples were deposited using a pulsed filtered cathodic vacuum arc (p-FCVA). A $20 \mathrm{~nm}$ Ti adhesion layer was first deposited by direct current magnetron sputtering (DC-MS). The other CNMs discussed in this study were grown by chemical vapor deposition (CVD) or plasmaenhanced chemical vapor deposition (PECVD). Prior to CNM growth, the adhesion and metal catalyst layers were deposited either with a continuous current arc, p-FCVA, radio frequency magnetron sputtering (RF-MS), or thermal evaporation. Further details can be found in Table 1.

Carbon nanofiber (CNF), and multi-wall carbon nanotube (MWCNT) were grown directly on Si wafers from physical vapor deposition (PVD) predeposited catalyst layers. Carbon nanowall (CNW) samples were grown from pyrolytic graphite placed on top of the Si substrate. Graphene ${ }_{C V D}$ was grown on copper foil and transferred to a Si wafer by first spin-coating poly(methyl methacrylate) (PMMA) onto the graphene/Cu film. An aqueous solution of $\mathrm{NaOH}$ was employed for electrochemical delamination (bubbling transfer) as an electrolyte, while $\mathrm{Cu}$ was polarized at $-3 \mathrm{~V}$ to delaminate PMMA/graphene. Then, PMMA/graphene was picked up by a carrier wafer to deionized water and transferred onto Si wafers, which were cleaned beforehand in acetone + ultrasonication for $5 \mathrm{~min}$ and rinsed in isopropyl alcohol.

Graphene $_{\mathrm{HT}}$ films were grown by annealing the Si face of 4$\mathrm{H} \mathrm{SiC} \mathrm{substrates} \mathrm{at} \mathrm{atmospheric} \mathrm{pressure} \mathrm{in} \mathrm{Ar} \mathrm{ambient} \mathrm{at}$ $1650{ }^{\circ} \mathrm{C}$ for $5 \mathrm{~min}$. Atomic force microscopy (AFM) measurements show a surface structure with a periodical terrace characteristic to graphene layers. The height of the terraces was around $0.5 \mathrm{~nm}$. The thickness and quality of the film were estimated by means of Auger and Raman spectroscopy that confirmed the presence of a single layer of graphene and low defect density in the graphene film before patterning. According to Auger measurements, the total coverage of the $\mathrm{SiC}$ surface by graphene was a bit less than 1. Indicating that the growth process was terminated before the second graphene layer started to grow. ${ }^{55}$

Single-wall carbon nanotube (SWCNTs) were synthesized by aerosol CVD and collected on nitrocellulose filters. The graphene and SWCNTs were press-transferred on $\mathrm{Si}$ substrates. More detailed descriptions of the deposition processes can be found based on the references given in Table 1.

Data Collection, Preparation, and Peak Fitting. XAS measurements were carried out at the Stanford Synchrotron Radiation Lightsource (SSRL) bending magnet beamline 8-2 at a $55^{\circ}$ incidence angle (magic angle) of X-ray incidence. Beamline $8-2$ is equipped with a spherical grating monochromator, operated using $40 \times 40 \mu \mathrm{m}^{2}$ slits, resulting in a resolution of around $200 \mathrm{meV}$. The spot size at the interaction point was around $1 \times 1 \mathrm{~mm}^{2}$ and the total flux was in the order of $10^{10}$ photons/s for which beam damage was not noticeable even for extended exposure. The X-ray energy for the carbon $1 \mathrm{~s}$, nitrogen $1 \mathrm{~s}$, and oxygen $1 \mathrm{~s}$ edges were scanned from 260 to 350,380 to 430 , and 520 to $560 \mathrm{eV}$, respectively. The data were collected both in the total electron yield (TEY) and Auger electron yield (AEY) modes using the drain current (amplified by a Keithley picoampmeter) and a cylindrical mirror analyzer (CMA) operated with a pass energy of $200 \mathrm{eV}$ and set to record the main Auger line for the various edges, respectively. The incoming flux was recorded using a nickel 


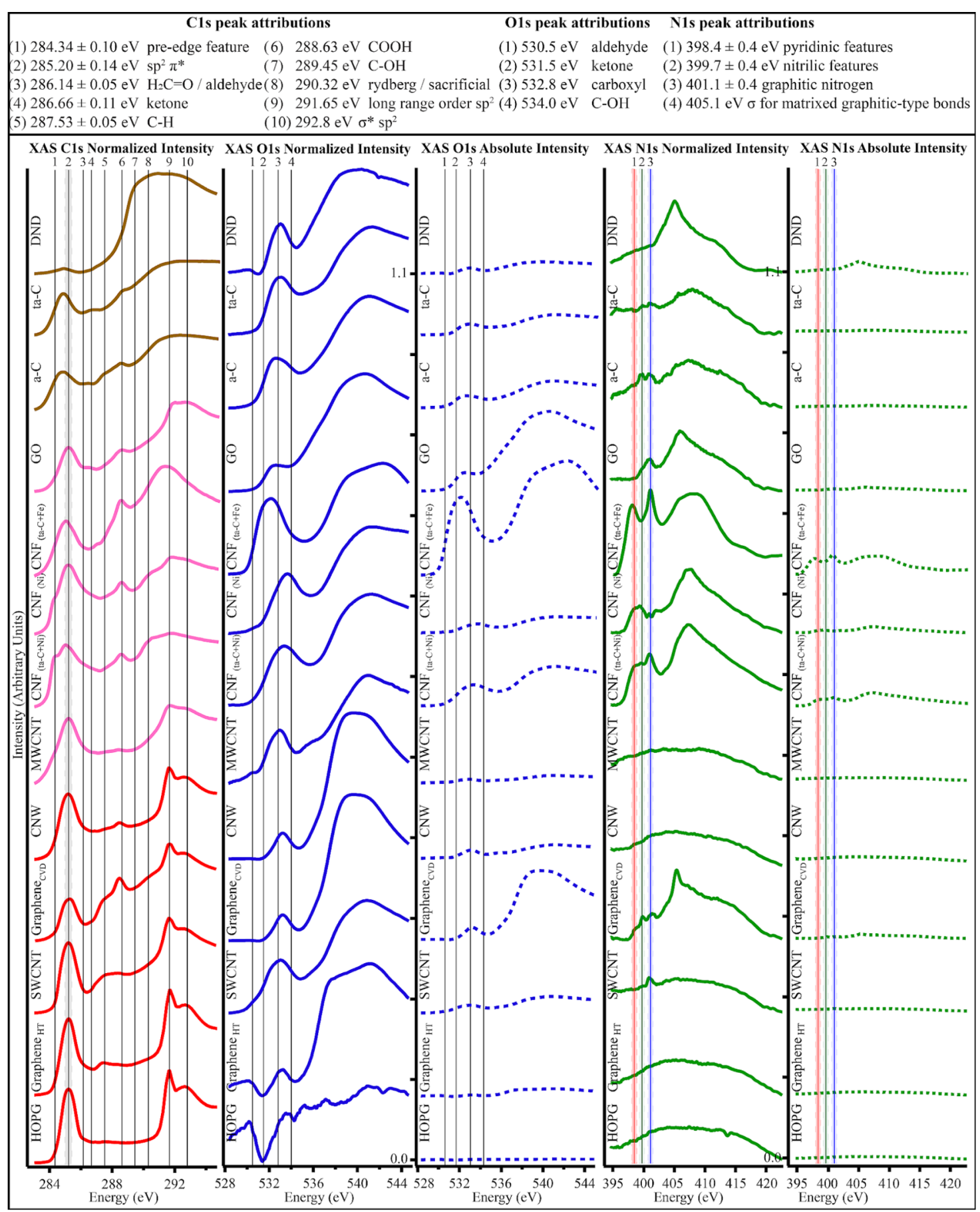

Figure 1. Trends in C 1s, O 1s, and N 1s XAS of 12 different carbon nanomaterials accompanied by highly ordered pyrolytic graphite (HOPG). Left is the $\mathrm{C} 1 \mathrm{~s}$ normalized spectra (red), next on the right is $\mathrm{O} 1 \mathrm{~s}$ normalized spectra (blue), center is $\mathrm{O}$ 1s absolute intensity spectra (blue dashed), next on the right $\mathrm{N} 1$ s normalized intensity (green), and on the right $\mathrm{N}$ 1s absolute intensity (green dashed). Peak positions are marked in the top legend and numbered and dashed lines drawn to the position of the expected peak positions to the spectra to guide the eye. Key for abbreviations: single-wall carbon nanotube (SWCNT), carbon nanowall (CNW), multi-wall carbon nanotube (MWCNT), carbon nanofiber (CNF), graphene oxide (GO), amorphous carbon (a-C), tetrahedral amorphous carbon (ta-C), and detonation nanodiamond (DND). Absolute intensity refers to the intensity that has not been normalized, meaning that the spectra have been background corrected and energy calibrated, but its intensity is as measured. Note that HOPG-normalized O 1s intensity features are likely caused by the normalization error as the absolute intensity is featureless.

grid with a Au sputtered film (called the i0). The data collected using the i0 follows the same procedure as the TEY measurements mentioned above. See Figure S1 for i0 spectra for $\mathrm{C} 1 \mathrm{~s}, \mathrm{O} 1 \mathrm{~s}$, and $\mathrm{N} 1 \mathrm{~s}$ edges. For details on how to carefully plan XAS measurements and for general information about the measurements, we refer the reader to refs 64-66.
The data were normalized as follows. The slope of the linear background (initially fitted to the pre-edge region) was allowed to vary within a small range to keep both the area and edge jump constant across the dataset. The theoretical underpinning of edge jump normalization is well established (textbook normalization) and represents the atomic cross-section for the 
atom, which is independent of the chemical surrounding (when done at high enough energy above the edge). ${ }^{64}$ The area normalization is based on the sum rules that associate the $\mathrm{X}$-ray absorption total cross-section with the density of unoccupied $2 \mathrm{p}$ states (in the case of a $1 \mathrm{~s}$ absorption even under dipole conditions). By forcing both the edge jump and the area to be the same, we are making the assumption that the difference in unoccupied $2 p$ states between the spectra is not varying, which is strictly not true when looking at different chemical environments. However, in practice, the knowledge about the exact background function to use in the textbook normalization (which is presumed in the edge jump) in the soft X-ray regime is in many cases weak, at best, and in many cases erroneous, resulting in wide variations in normalization across similar samples depending on the signal to background and other experimental conditions, a highly undesirable condition. Making use of a stricter normalization rule such as keeping the area and edge jump constant will result in a very robust collective dataset that can be used with much higher confidence to look at small variations between similar samples, and it avoids experimental conditions and normalization artifacts to play a significant role in the analysis. Moreover, it provides a transparent way of comparing spectra across studies. Thus, we have applied this method here.

The data was prepared for publication using IGOR 8.06 software. Prior to the peak fitting procedure, the data was energy corrected and aligned using a Gaussian peak fit to the $\mathrm{C}$ $1 \mathrm{~s} \mathrm{sp}^{2} \pi^{*}$ feature of the reference sample and shifting all data accordingly to match the C $1 \mathrm{~s} \mathrm{sp}^{2} \pi^{*}$ at $285.2 \mathrm{eV}$. Furthermore, $\mathrm{C} 1 \mathrm{~s}, \mathrm{O} 1 \mathrm{~s}$, and $\mathrm{N} 1 \mathrm{~s}$ regions were area normalized over the $\pi$ and $\sigma$ regions. Area normalization was performed (energy ranges of 280-320, 395-425, and 527$547 \mathrm{eV}$ was set to have an area of 20) to enable comparison of relative intensities within the dataset.

A custom peak fitting procedure was developed using asymmetric Gaussian functions for sharper features corresponding to bonded states below the ionization potential (IP), broader asymmetric line-shapes that captures sigma states, two ionization potentials, and a smoothed background HOPG signal to capture $\mathrm{sp}^{2}$-like carbon (see Figure $\mathrm{S} 2$ in the Supporting Information for more details). All of the Gaussian functions representing different functional groups were assigned to have a maximum full width at half-maximum (FWHM) of 0.9 to attempt to capture specific locations of functional groups in the energy regime. Prior to fitting, the energy range in the $\mathrm{C} 1 \mathrm{~s}$ was given variable weight, 1 for $280-$ 283.2 and $292.25-309.2 \mathrm{eV}, 5$ for $283.25-292.2$, and 0.1 for 309.25-320 eV. The motivation for these choices is discussed in the Results and Discussion section.

The data displayed in Figures 2 and 3 show a clear shift in the C $1 \mathrm{~s} \mathrm{sp}^{2} \pi^{*}$ energy, which is considered in detail in the next section. The energy calibration from the reference sample dip was further confirmed by carefully observing the alignment of the excitonic signature at $291.65 \mathrm{eV}$ (which matches the literature value of $291.65 \mathrm{eV}^{66-68}$ very well), where present. If the excitonic feature was not visible (which is a strong indication of the material's lack of long-range order), the carboxyl reference value was used at $288.63 \mathrm{eV}$, as the carboxyl feature is stable and well defined in energy, as proven both computationally ${ }^{69}$ and experimentally. ${ }^{27}$ The reference spectra for $\mathrm{sp}^{3}$ contribution were aligned to match the second order bandgap of the diamond at $302.5 \mathrm{eV}$, which is clearly present in both the extracted literature reference spectra and the DND sample (see Figure S3).

The exciton in this study refers to graphitic $\left(\mathrm{sp}^{2}\right)$ exciton at energy $291.65 \mathrm{eV},{ }^{66-68,70}$ and it should not be mixed with diamond $\left(\mathrm{sp}^{3}\right)$ bulk exciton at energy $289.30 \mathrm{eV}^{37,71}$ or with the $\sigma^{*}$ transition, near $292.3-293.0 \mathrm{eV} .^{66,70,72-74}$ Additionally, the long-range order description of this exciton is used interchangeably with the excitonic signature as it describes the degree of order in the $\mathrm{sp}^{2}$ matrix. As can be clearly seen in Figure 1 left column where $\mathrm{C} 1 \mathrm{~s}$ spectra for the carbon nanomaterials are shown, the amount of $\mathrm{sp}^{2}$ bonding (the intensity of the $\mathrm{sp}^{2}$ peak at $285.20 \mathrm{eV}$ ) does not correlate with the excitonic feature at $291.65 \mathrm{eV}$. However, there is a marked correlation between the position and shape of the $\mathrm{sp}^{2} \pi^{*}$ peak and the appearance of the excitonic feature, as discussed in the Results and Discussion section.

As the exciton is not a chemical state, it is not driven by the changes in the local density of states (DOS) around the excited site. The carbon network allows the excitonic feature to form in the core-hole state and does not have a direct correspondence with the DOS. It is true that there is some directionality of the excitonic feature that makes it better visible at a higher incidence angle, but it does not broaden under any circumstances. Chemical states, in particular $\sigma$-like states and bands, will be shifted in energy due to a spread of carbon-carbon distances that occur with the increased amount of disorder. Disorder, resulting in broader sigma bands and states, is correlated with the disappearance of the exciton, but the exciton does not change in energy and width, reflecting the different characteristics of the exciton and sigma features. Generally, states in the $\pi^{*}$ region are more stable in energy since they are primarily driven by the local coordination, and the states are also not protruding far out from the excitation center making them less likely to rehybridize in the presence of second and third shell changes. Some states with a particularly local character, such as the carbonyl $\pi^{*}$ in the carboxyl group, are almost unaffected by the surrounding disorder.

Computational Studies. Computational methods offer new opportunities for studying and interpreting the experimental results. Computational ab initio references are free from the bias and controversy present in the literature references. The accuracy of the method depends on the level of theory that is used as well as the size of the dataset. Three total energy calculations are needed to obtain the spectra: two to find the right energy alignment and one to obtain the shape of the spectrum. In addition, the geometry and the electronic structure of the models have to be relaxed. The results presented here consist of hundreds of spectra (548 exactly), and the depicted spectra (Figure 2) are the average over all of the sites in the structures (Figure 3). In this case, the computational results seem to be in good agreement with experimental interpretation. The simulations were carried out at the density functional theory (DFT) level by utilizing the GPAW code. ${ }^{75,76}$ The functional that was used was PerdewBurke-Ernzerhof (PBE), ${ }^{77}$ and van der Waals corrections were included. ${ }^{78}$ Calculations were performed under periodic boundary conditions. Further details of the computational method can be found in refs 69 and 79-81.

\section{RESULTS AND DISCUSSION}

In the following, we will discuss the soft $\mathrm{x}$-ray spectroscopy results originating from the total electron yield (TEY) spectra unless otherwise specified. The spectra shown in Figure 1 


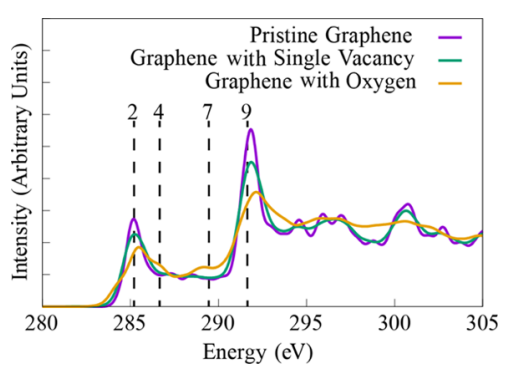

Figure 2. Origin of the broadening of $\mathrm{sp}^{2} \pi^{*}$ and other features of the spectra explained by utilizing density functional theory (DFT) simulations. Pristine graphene displays the same trend as HOPG, where there are no peaks indicating the presence of oxygen between the $\operatorname{sp}^{2} \pi^{*}$ peak and the $\sigma^{*}$ peaks. The numbering used here for the spectral features is the same as used in Figure 1.

reveal the $\mathrm{C} 1 \mathrm{~s}$ normalized intensities and for $\mathrm{O} 1 \mathrm{~s}$ and $\mathrm{N} 1 \mathrm{~s}$, both their absolute (where absolute intensity refers to the intensity that has not been area normalized but is shown as measured with i0 normalization) and normalized intensities (all materials were fabricated for this study, see Table 1 in the Experimental Section for details). Based on the C 1s, O 1s, and $\mathrm{N}$ 1s spectra shown in Figure 1, it is clear that all carbon nanomaterials studied have some oxygen contamination. The only material that appears nearly oxygen free is the highly ordered pyrolytic graphite (HOPG) reference sample as its C $1 \mathrm{~s}$ spectra have peaks only at the well-established $\pi^{*}(285.2$ $\mathrm{eV}$ ) and core exciton $291.65 \mathrm{eV}$ from the $\mathrm{sp}^{2}$ network and no peaks between them associated with chemically shifted functional groups that would be present otherwise.

We have concentrated our $\mathrm{O} 1 \mathrm{~s}$ analysis efforts within the $\pi$ region (below $\sim 535 \mathrm{eV}$ ) as the intensities observed in the $\sigma$ region could be arising from native oxide present at the $\mathrm{Si}$ wafer surface, which was used as a substrate for all of the carbon nanomaterials in this study. Based on the full dataset, the degree of the surface oxygen contamination changes drastically among the various carbon allotropes. Based on the TEY spectra, the most oxygen-rich surface is observed on tetrahedral amorphous carbon $(\mathrm{ta}-\mathrm{C})+\mathrm{Fe}$ grown carbon nanofibers (CNFs), whereas the least amount of oxygen is observed on high temperature (HT) grown graphene (except for the reference HOPG, which has nearly no oxygen). These results are consistent with the more surface-sensitive Auger electron yield (AEY) study, which indicates strong agreement with the findings from the TEY study.

In our earlier work, ${ }^{45,82}$ we fitted the $\mathrm{C} 1$ s region with the following peaks: $\operatorname{sp}^{2} \pi^{*}, \mathrm{C}-\mathrm{OH}$, ketone/aldehyde groups, carboxyl, (Rydberg states just below the ionization potential (IP)) and the long-range order ( $\mathrm{sp}^{2}$ core exciton). With this large dataset, it was not feasible to find transferrable fitting parameters, i.e., peak positions that can be used to fit the spectra of all of the materials, without significant peak travel (over $\pm 300 \mathrm{meV}$ ). Thus, additional peaks were fitted between the $\operatorname{sp}^{2} \pi^{*}$ and the exciton peaks to match the observed intensity. Furthermore, it is of high importance to note that the energy assignments of the carbon-oxygen functional groups in this work are approximations and are used to investigate trends in this dataset. Additionally, due to the differences in the properties of the carbon matrix between different samples, the $\mathrm{sp}^{2} \pi^{*}$ peak has been allowed to float at $285.25 \pm 0.15 \mathrm{eV}$. To capture the differences between materials that have welldefined and sharp $\mathrm{sp}^{2} \pi^{*}$ transition and those with a broader $\mathrm{sp}^{2} \pi^{*}$ feature, an additional peak was added to capture this intensity. This peak has been placed at $284.3 \pm 0.1 \mathrm{eV}$. The positions for each functional group have been taken from the literature. ${ }^{27,29,30,37,64,72,73,83-91}$ We acknowledge that the oxygen functional group assignment shows poor agreement across many publications in material science. The most consistent information can be found from the electron energy loss spectroscopy (EELS) studies of molecular compounds measured by Hitchcook and co-workers ${ }^{29}$ as well as for carboxyl groups, which are well assigned and will be discussed later. Our logic for peak assignments follows closely that of Urquhart and $\mathrm{Ade}^{21}$ (and citing work ${ }^{27,29,30,37,64,83-90}$ ) in which chemical shifts for a number of different carbonyl groups were addressed systematically. For functional groups that are not readily available from simple molecular compounds, there is an inherent difficulty to assign spectral features and reliable and unbiased energy assignment is difficult to obtain. ${ }^{21,29,30}$ Thus, using computational data to support the selection of peak positions would be extremely helpful. For carbon nanomaterials, this issue has been tackled in a recent work by Aarva et al., ${ }^{69,92}$ where the features present in the $\mathrm{C} 1 \mathrm{~s}$ spectra of amorphous carbon thin films have been calculated from individual structural fingerprints where computationally generated structural models were sampled. However, there are computational studies that do not directly support our findings, such as Hunt et al. ${ }^{93}$ Especially when the matrix is
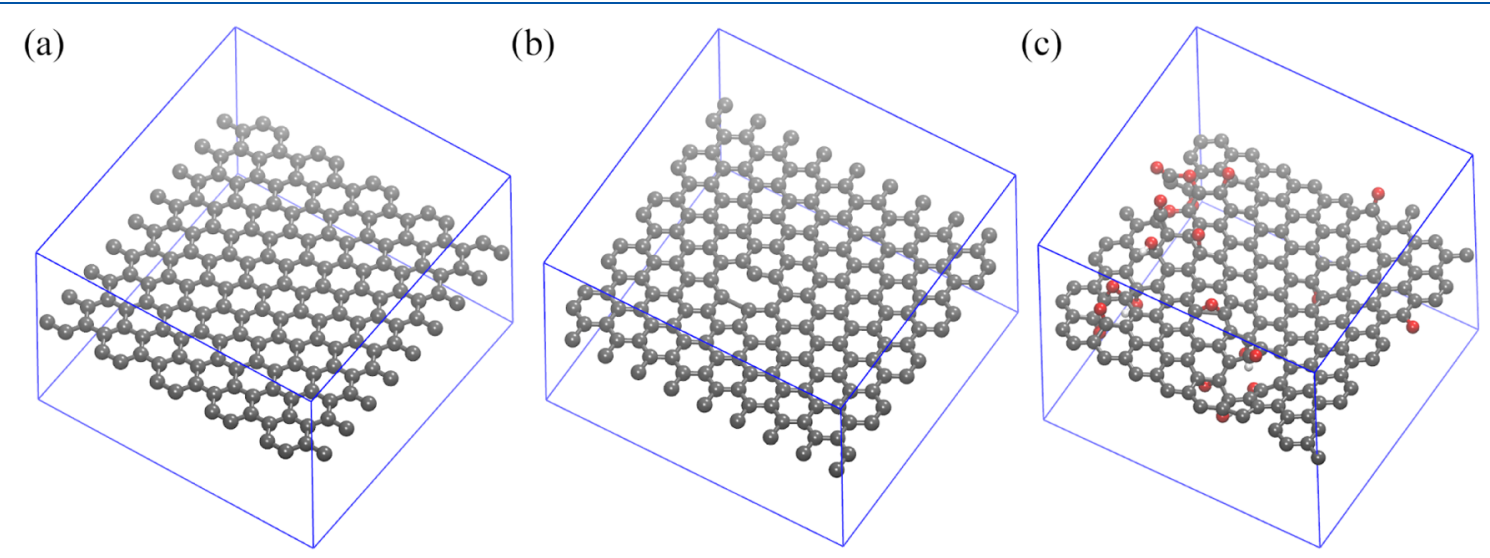

Figure 3. Structures that were used in simulations: (a) pristine graphene, (b) graphene with a single vacancy, and (c) graphene with oxygencontaining groups. The oxygen-containing structure (c) has been originally published in ref 113 . 
Table 2. Normalized Intensities Extracted from the C 1s Spectra of Different Carbon Nanomaterials Sorted by Their Representative Groups in the Same Order as in Figure $1^{a}$

\begin{tabular}{|c|c|c|c|c|c|c|c|c|c|c|c|c|c|c|c|}
\hline & 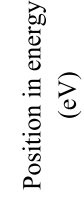 & $\begin{array}{l}\bar{D} \\
\mathbb{J}^{ \pm}\end{array}$ & 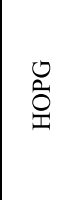 & 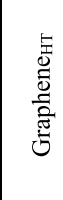 & 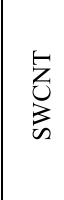 & 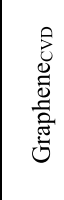 & $\sum_{3}^{3}$ & $\stackrel{\text { 乬 }}{\sum_{\Sigma}}$ & 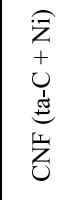 & $\underset{\mathrm{Z}}{\stackrel{\Xi}{Z}}$ & 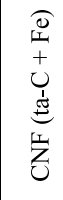 & O & 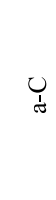 & せٕ & 方 \\
\hline $\mathrm{sp}^{2}$-pre & 284.34 & 0.10 & 0.01 & 0.01 & 0.01 & 0.04 & 0.02 & 0.18 & 0.34 & 0.21 & 0.42 & 0.09 & 0.27 & 0.28 & 0.01 \\
\hline $\mathrm{sp}^{2}$ & 285.20 & 0.14 & 0.60 & 0.37 & 0.38 & 0.12 & 0.14 & 0.17 & 0.30 & 0.29 & 0.43 & 0.10 & 0.06 & 0.17 & 0.04 \\
\hline $\mathrm{H}_{2} \mathrm{C}=\mathrm{O} /$ aldehyde & 286.14 & 0.05 & 0.07 & 0.05 & 0.02 & 0.01 & 0.02 & 0.10 & 0.24 & 0.19 & 0.23 & 0.00 & 0.03 & 0.06 & 0.01 \\
\hline ketone & 286.66 & 0.11 & 0.08 & 0.07 & 0.03 & 0.03 & 0.07 & 0.09 & 0.18 & 0.15 & 0.17 & 0.08 & 0.11 & 0.13 & 0.01 \\
\hline $\mathrm{C}-\mathrm{H}$ & 287.53 & 0.05 & 0.14 & 0.20 & 0.27 & 0.35 & 0.12 & 0.18 & 0.32 & 0.27 & 0.55 & 0.16 & 0.30 & 0.22 & 0.01 \\
\hline carboxyl & 288.63 & 0.00 & 0.10 & 0.15 & 0.26 & 0.54 & 0.18 & 0.20 & 0.47 & 0.43 & 0.89 & 0.29 & 0.38 & 0.34 & 0.15 \\
\hline rydberg & 289.45 & 0.00 & 0.03 & 0.05 & 0.11 & 0.21 & 0.08 & 0.12 & 0.24 & 0.20 & 0.52 & 0.14 & 0.20 & 0.18 & 0.26 \\
\hline $\mathrm{ryd} / \mathrm{sac}$ & 290.32 & 0.00 & 0.02 & 0.08 & 0.02 & 0.06 & 0.08 & 0.01 & 0.05 & 0.06 & 0.12 & 0.01 & 0.01 & 0.01 & 0.01 \\
\hline $\mathrm{sp}^{2}$ long range & 291.65 & 0.00 & 0.32 & 0.19 & 0.10 & 0.06 & 0.09 & 0.01 & 0.00 & 0.00 & 0.03 & 0.03 & 0.00 & 0.00 & 0.00 \\
\hline $\mathrm{sp} 2$ & & & 0.49 & 0.60 & 0.56 & 0.48 & 0.78 & 0.73 & 0.56 & 0.68 & 0.45 & 0.52 & 0.45 & 0.31 & 0.00 \\
\hline sp3 & & & 0.00 & 0.00 & 0.00 & 0.00 & 0.00 & 0.00 & 0.00 & 0.00 & 0.00 & 0.00 & 0.00 & 0.11 & 0.49 \\
\hline $\mathrm{sp}^{2} \%$ & & & 1.00 & 1.00 & 1.00 & 1.00 & 1.00 & 1.00 & 1.00 & 1.00 & 1.00 & 1.00 & 1.00 & 0.73 & 0.00 \\
\hline $\mathrm{sp}^{3} \%$ & & & 0.00 & 0.00 & 0.00 & 0.00 & 0.00 & 0.00 & 0.00 & 0.00 & 0.00 & 0.00 & 0.00 & 0.27 & 1.00 \\
\hline
\end{tabular}

${ }^{a}$ The depth of green shows which of the functional groups has the highest intensity in the given material (the darker the color, the higher the intensity).

complex, traditional peak fitting schemes are quite inadequate for capturing all of the spectral features from functional groups, and its feasibility is thus inherently limited for many carbon nanomaterials. Finally, the $\mathrm{sp}^{2}$ and $\mathrm{sp}^{3}$ ratios for each material were extracted using a smoothed HOPG reference as a material to describe the $\mathrm{sp}^{2}$ contribution (which is discussed in more detail below) and the CVD grown $\mathrm{sp}^{3}$ containing film ${ }^{94-96}$ as the measure of $\mathrm{sp}^{3}$ bonding. These CVD-grown films were grown in a hydrogen environment, and we selected the "H-450 W" from Coffman et al. work as the most representative for describing the $\mathrm{sp}^{3}$ bonding in materials of this study. This is due to the fact that the sharp diamond exciton observed at $289.3 \mathrm{eV}$ in the literature ${ }^{71,87,94}$ is absent from all materials investigated in this study, including also the ones that are most likely to exhibit a significant amount of $\mathrm{sp}^{3}$ bonded carbon, namely, in tetrahedral amorphous carbon (taC) and detonation nanodiamond (DND) samples, as can be seen from Figure 1 (see also Figure S3 for comparison of ta-C and DND with the $\mathrm{H}-450 \mathrm{~W}$ reference spectra). The proposed method to extract the $\mathrm{sp}^{2}$ and $\mathrm{sp}^{3}$ ratios does not take into account that (i) some of the intensities at peaks at 284.34 and $285.2 \mathrm{eV}$ are likely arising from the $\mathrm{sp}^{2}$ carbon and (ii) the $\mathrm{sp}^{3}$ background does not capture all $\mathrm{sp}^{3}$-bonded carbon, especially in the case of amorphous diamond-like carbon (ta-C and possibly to some degree also a-C like samples). The literature has some authoritative work, e.g., ref 71 and 94, studying how the changes in the carbon matrix $\left(\mathrm{sp}^{3}\right.$ vs $\left.\mathrm{sp}^{2}\right)$ changes their representative XAS spectra, but there is no fitting procedure proposed. There are some publications about the fitting procedure for extracting the $\mathrm{sp}^{2}$ ratio using the $\mathrm{sp}^{2} \pi^{*}$ peak and integrating over the energy range to capture the $\mathrm{sp}^{2} \sigma^{*}$ intensity, ${ }^{97}$ but a clear debate exists on the selection of the appropriate $\sigma^{*}$ energy range. ${ }^{98-101}$ Additionally, not all of the observed changes in the spectra have been strongly connected to the structural changes; therefore, the differences between the carbon matrix containing crystalline, partially crystalline, and amorphous $\mathrm{sp}^{3}$ carbon are hard to compare with respect to their $\mathrm{sp}^{3}$ content. This calls for further experimental and computational work to produce strong fingerprints for both amorphous and crystalline $\mathrm{sp}^{3}$ materials with and without oxygen contaminants. Thus, there are clear similarities in ta-C and a-C spectra (see Figure 1), but the fit suggests that there is no $\mathrm{sp}^{3}$ in $\mathrm{a}-\mathrm{C}$, and the result needs to be critically evaluated.

In principle, computational tools can provide a new route toward establishing more robust links between the atomic motifs present in the materials and the corresponding spectral features found on their X-ray spectra. The accuracy of these tools can be validated against molecular refs 102-104 and ideal extended structures (e.g., graphene, HOPG, and diamond), and then used to accurately predict and interpret the experimental spectra of more complex materials. ${ }^{69,92}$ For instance, computationally, one can establish a one-to-one correspondence between each of the functional groups present on a material and their contribution to the spectra throughout the full range of scanned energies. However, since the required and reliable computational structural models are not yet available for fitting the whole dataset used in this study (see Aarva et al. ${ }^{69,92}$ ), we were forced to proceed with traditional peak fitting to retain the consistency over the whole dataset. Instead, we use computational support to understand certain features that are not readily accessible from the literature, such as the peaks seen well below $285 \mathrm{eV}$ in $\mathrm{C} 1$ s spectra of 

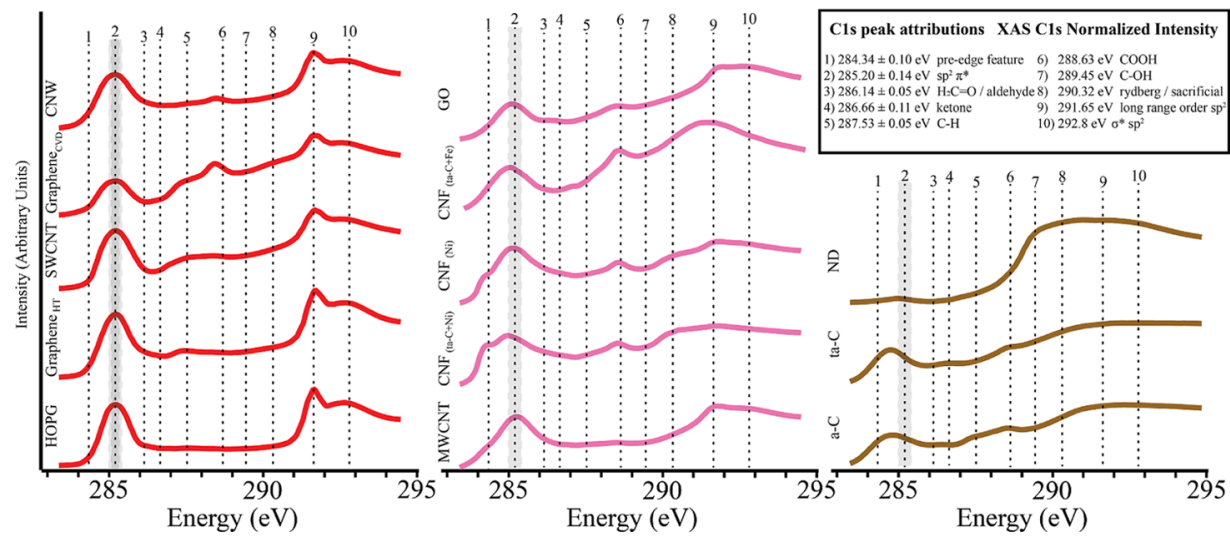

Figure 4. C 1s spectra are arranged into three different subgroups, reading from the left, (red) we see the materials with clear crystalline, long-range order; in the middle, (pink) we see the materials mixed with some existing long-range order, but visible broadening of the $\mathrm{sp}^{2} \pi^{*}$ features; and on the right, (brown-green) we see amorphous materials and ND with no clear presence of the $\mathrm{sp}^{2}$ long-range order and either nonexistent or broadened and shifted (to lower energy) $\mathrm{sp}^{2} \pi^{*}$ features.

amorphous carbon (a-C) and ta-C. An additional note on the analysis of the $C 1$ s region is that we expect, based on the earlier studies ${ }^{27,72,88,105-110}$ and the $\mathrm{N}$ 1s spectra recorded during this study (see Figure 1), that some of the features presently attributed to carbon-oxygen functional groups are in reality due to carbon-nitrogen bonds. However, as the amount of nitrogen present in these carbonaceous materials is very low (only the CNF and DND show any significant signal in the $\mathrm{N}$ $1 \mathrm{~s}$ scans) and the analysis of the $\mathrm{O} 1 \mathrm{~s}$ spectra corroborates much of the functional group assignment in the $\mathrm{C} 1 \mathrm{~s}$ region (the carbon-oxygen functional group assessment), the relative contribution of intensity from nitrogen functionalities in the $\mathrm{C}$ 1s spectrum is expected to be small, and we cannot expect to extract any meaningful conclusions about the nature and prevalence of nitrogen functional groups from the $\mathrm{C} 1 \mathrm{~s}$ spectra. The results from the fitting are summarized in Table 2.

The two IP positions (from HOPG and a separate ramp function to describe individual ionization of the oxygen functional groups, both of which will be discussed in more detail shortly and shown in Figure S4 in the Supporting Information) were selected to provide more rigorous and reasonable fitting results in the energy regime directly after the $\operatorname{sp}^{2} \pi^{*}$ and before the Rydberg states. Here, we used HOPG as a reference material whose surface has very little oxygen, as can be seen from the right column $\mathrm{O}$ 1s spectra in Figure 1. Importantly, the intensity increase after the $\pi^{*}$ feature on the HOPG C 1 s spectra is not arising from oxygen functionalities. Instead, the observed increase is caused by the metallic-type behavior of the pure carbon matrix as described in detail in the literature and also shown in this work (no counts were collected while scanning the $O$ 1s region). ${ }^{66,67,91,111}$ Furthermore, as HOPG is widely regarded in the literature as a "gold standard" carbon reference, we selected the HOPG spectra as a carbon-background after smoothing it with a 20point moving-average procedure. This background was used for all $\mathrm{sp}^{2}$ containing materials (which ruled out DND). This background selection also represents the IP of the pure carbon matrix and the possible Rydberg intensities for pure carbon. Additionally, an $\mathrm{sp}^{3}$ background was introduced to the fit, which like the $\mathrm{sp}^{2}$ background and all of the fit in general, was let to find its amplitude through the fit by minimizing the $\chi^{2}$ test. To further improve the fit and to represent the IP for each individual oxygen functional group, a ramp function was fitted starting directly after the $\mathrm{H}_{2} \mathrm{C}=\mathrm{O}$ /aldehyde with a slope of 1 /
3 (similar representation of the continuum of the $\pi^{*}$ states is used by Jiménez et al. ${ }^{105}$ ). It should be noted that in this study, the higher energy transitions in the $\sigma$ region are not of interest due to such a large array of functional groups at the carbon nanomaterial surface.

Both of the graphene samples in this study are monolayers (see the Experimental Section and Figure S5, for graphene ${ }_{\mathrm{CVD}}$ sample details). Thus, in the total electron yield mode, we can expect some contribution from the substrate. The wafers used in this work are likely to have a thin native oxide film of $\mathrm{SiO}_{x}$ on their surface as well as some carbon and oxygen contamination on the wafer surfaces that cannot be ignored. However, as can be seen in the graphene ${ }_{\mathrm{HT}}$ spectrum, which closely matches the literature references in the absence of any significant intensity in the region between the $\mathrm{sp}^{2} \pi^{*}$ and the $\mathrm{sp}^{2}$ core exciton, see, e.g., refs 68,72 , and 112 , the contributions of the other carbon species to the $\mathrm{C} 1 \mathrm{~s}$ spectrum is at most very small. However, as discussed above, it is still likely that some of the oxygen intensities observed are arising from the silicon wafer. Especially for the graphene ${ }_{\mathrm{HT}}$ case, the relatively high intensity in the $\mathrm{O} 1 \mathrm{~s}$ post- $\pi$ region compared to the pre- $\sigma$ region can be partly attributed to the featureless $\mathrm{SiO}_{x}$ contribution. Nevertheless, in general, we note that the absolute $\mathrm{O} 1 \mathrm{~s}$ signal is very low. Thus, both the $\mathrm{O} 1 \mathrm{~s}$ and $\mathrm{C}$ 1s spectra indicate that the contribution from carbonaceous contaminants does not play a major role in the analysis, especially for the pre- $\sigma$ region. The graphene ${ }_{\mathrm{CVD}}$, on the other hand, has a clear carboxyl peak at $288.63 \mathrm{eV}$ and also a clear peak in the $\pi$ region of the $\mathrm{O} 1 \mathrm{~s}$ spectra, indicating higher oxygen loading than for the graphene $\mathrm{HT}_{\mathrm{HT}}$.

Next, we briefly discuss the computational results and their connection to the experimental data and especially use them to interpret the low energy features observed in the $\mathrm{C} 1 \mathrm{~s}$ spectra of a-C and ta-C. We are particularly concerned by the effect on the $\mathrm{C}$ 1s spectrum from added disorder and the introduction of other elements, such as oxygen. The computational spectra shown in Figure 2 highlight the drastic differences between pristine graphene, graphene with one vacancy per unit cell (see Figure 3), and graphene with oxygen-containing groups (the oxygenated structure is obtained from ref 113). The structures used in the simulations are visualized in Figure 3. The dashed lines in Figure 2 indicate the same $C$ 1s peak attributions, with the numbering used consistently throughout this work (see Figure 1). Only relevant peak positions are depicted: (1) $\mathrm{sp}^{2}$ 
$\pi^{*}$, (4) ketone, (7) $\mathrm{C}-\mathrm{OH}$, and (9) long-range order $\mathrm{sp}^{2}$. Each spectrum is shifted by $0.55 \mathrm{eV}$ on the energy scale to match the $\mathrm{sp}^{2} \pi^{*}$ peak position. Based on these quantitative results, it is clear how the features of the spectra are broadened and spectral features at low energies emerge after a defect or other elements are introduced to the pristine ordered system. As discussed in detail in our previous work, ${ }^{92,114}$ atomic-level structural models of the materials under investigation are required for constructing the XAS spectra from first principles. To have enough statistics to represent the real, nonideal materials adequately, a statistically significant sampling of computationally generated structures is required. ${ }^{92,114}$ For liquids and gases, this sampling is favorably done via molecular dynamics (MD), but this approach is not applicable for disordered solids (unless they can be treated as glasses). Since a statistically significant database of all relevant allotropes of carbon used here is outside of the scope of this study, we decided to use traditional fitting procedures for all of the structures investigated.

To further investigate the overall trends in the C 1s region, we have arranged the spectra into three distinctive groups based on the form that the carbon network takes (depending on their $\mathrm{sp}^{2} \pi^{*}$ intensity, peak width, and position in energy) and the presence of the long-range order. The three different distinctive groups are crystalline, mixed, and amorphous, as shown in Figure 4. All of the $\mathrm{sp}^{3}$ carbon containing materials also fall into the latter category.

This classification clearly shows that any material found in this study having a long-range order in the $\mathrm{sp}^{2}$ matrix will also have a clear and sharp $\mathrm{sp}^{2} \pi^{*}$ feature. The broader $\mathrm{sp}^{2} \pi^{*}$ feature seems to strongly correlate with the lack of distinctive oxygen functionalities (no clear peak in the spectra) on most of the amorphous surfaces, as none of these materials have a clear peak present in the $C 1 \mathrm{~s}$ spectra. It is possible that the decreased surface area, flatter surface features, heterogeneous surface energetics, and contributions from the dissolved oxygen in the matrix could make it unfavorable for selective functional group bonding and/or formation at the surface of the amorphous materials presented in this study. Materials in the amorphous category also include at least some degree of $\mathrm{sp}^{3}$ bonding, which is another contributing factor in the local electronic structure. Thus, instead of some groups dominating the spectra, we will observe contributions from a wide variety of different groups. The effect of the bulk, which is dominant in thin films when compared to porous networks on, e.g., SWCNTs, MWCNTs, and aligned structures of CNW and $\mathrm{CNF}$, may lead to the signal from surface functional groups being overwhelmed by that arising from the bulk, as shown in our earlier work with ta-C. ${ }^{115}$

Furthermore, the materials with the clearest and most abundant presence of oxygen functional groups are those in the mixed category. Very interestingly, only carboxyl at $288.63 \mathrm{eV}$ is prominent enough to show a distinctive peak for any sample. When looking at the peak intensities after the fitting procedure, as shown in Table 2, carboxyl intensity is the dominant functional group for all carbon nanomaterials, except for graphene $_{\mathrm{HT}}$ and SWCNT, which both have low oxygen content on their surface. As we have shown earlier with computational results, ${ }^{69,92}$ the carboxyl functional group is the most stable (with regard to its peak position), regardless of the environment where the $\mathrm{COOH}$ group is bonded ( $\mathrm{sp}$ or $\mathrm{sp}^{2}$ site), always showing a distinctive fingerprint at the same energy. At the same time, carboxyl groups, due to their chemical structure, protrude higher from the surface of the material than other functional groups discussed here, as shown quantitatively in refs 92 and 114. This makes their energy less dependent on the local chemical environment than those of the other functional groups. There have been earlier reports describing different oxygen functional groups in different environments. One excellent example, showing spectra containing sharp features around $288.6 \mathrm{eV}$, is the work of Solomon et al. ${ }^{27}$ Additionally, there seems to be a consensus that the carboxyl peak lies in the 288.5-288.8 eV region, based on much of the $\mathrm{C} 1 \mathrm{~s}$ XAS literature. The computational results provided in refs 92 and 114 have now been able to provide the quantitative grounds for these earlier, more qualitative reports.

The $\operatorname{sp}^{2} \pi^{*}$ features observed, especially in Figure 4, reveal that both the position and the full width at half-maximum (FWHM) of the peak change depending on the material. For the crystalline materials, the $\mathrm{sp}^{2} \pi^{*}$ peak is sharp and quite well established at 285.2-285.3 eV. In addition, the excitonic feature at $291.65 \mathrm{eV}$ is also clearly visible with these materials. Especially with regards to the mix of crystalline and amorphous $\mathrm{sp}^{2}$ materials, namely, MWCNTs and all CNFs (which also include a higher amount of metals than the crystalline materials), there is a clear trend with the $\mathrm{sp}^{2} \pi^{*}$ peak appearing at lower energy and being broader than for the crystalline materials. This process is closely associated with the gradual disappearance of the $\mathrm{sp}^{2}$ excitonic feature from the spectra. Broadening of the $\mathrm{sp}^{2} \pi^{*}$ peak is expected to arise from the differences in the electronic structure of the $\mathrm{sp}^{2}$-rich matrix when both amorphous and crystalline $\mathrm{sp}^{2}$ are present. This was observed in our previous work, ${ }^{45,69,92}$ which can also explain the lower energy position of the $\mathrm{sp}^{2} \pi^{*}$ peak in the mainly amorphous films, as shown in Figure 2.

Interestingly, we also observe a shoulder feature in the $\mathrm{C} 1 \mathrm{~s}$ spectra (at $284.2 \mathrm{eV}$ ) of the $\mathrm{CNF}$, which have been grown using $\mathrm{Ni}$ or ta- $\mathrm{C}+\mathrm{Ni}$ catalyst layers. From these, the intensity of the lower energy peak of the doublet is more prominent in the ta-C $+\mathrm{Ni}$ grown CNF. As has been shown in ref 58, the two types of CNFs have different structures. The Ni grown CNF has a bamboo-like structure, where the ta-C + Ni CNF has a platelet-like structure. Studies on aromatic hydrocarbons have shown a correlation between the number of isolated double bonds and a lowering of the lowest unoccupied molecular orbital (LUMO), as observed in XAS spectra ${ }^{116,117}$ and even shown to induce separate split peaks in kinetically more unstable polycyclic compounds such as tetracene and pentacene $^{23}$ (on the opposite side of graphene). The lowering of the $\pi^{*}$ intensity therefore indicates the presence of more kinetically unstable $\mathrm{sp}^{2}$ configurations. Theoretical calculations also indicate that the lower the energy of the peak in the $\mathrm{C} 1 \mathrm{~s}$ spectra, the more reactive the site is expected to be. ${ }^{69}$ This indicates that the platelet-like structure would have more reactive sites than the bamboo-like fibers. Another possible explanation is discussed by Schultz et al., ${ }^{118}$ who reported splitting of the $\mathrm{sp}^{2} \pi^{*}$ feature with graphene grown on different metals $(\mathrm{Ni}, \mathrm{Cu}, \mathrm{Co})$. Graphene can produce two clearly distinct peaks in the $\mathrm{sp}^{2} \pi^{*}$ region when grown on polycrystalline $\mathrm{Ni}$ and has the ability to exhibit varying peak splitting depending on the detailed growth parameters. This is expected to arise from interactions between the metal and graphene lattice. This explanation is supported by findings from Huttmann et al., ${ }^{119}$ where graphene was intercalated with a monolayer of Eu. While still on the Ni film, only one peak was observed, indicating that the electronic structure of 
graphene is clearly affected by the metals it is exposed to. Thus, our observation of the shoulder feature in the two different $\mathrm{CNF}$ systems could be explained along these lines. In both CNF grown using $\mathrm{Ni}$, the resulting $\mathrm{CNF}$ has graphene-like sheets (either as a bamboo-like structure where the individual bamboo units are formed of graphene-like sheets in $\mathrm{Ni}$ grown $\mathrm{CNF}$ or stacked graphene sheets like in the ta-C $+\mathrm{Ni}$ grown $\mathrm{CNF}$ ) and the energy difference of the $\mathrm{sp}^{2} \pi^{*}$ shoulder features could be arising from the differences between the $\mathrm{Ni}-\mathrm{C}$ interface and interactions in the two distinct structures. Furthermore, since both $\mathrm{Ni}$ layers were deposited using different methods (in the Ni grown CNF sputtered and in ta-C $+\mathrm{Ni}$ grown $\mathrm{CNF}$ using a filtered cathodic vacuum arc (FCVA)), the microstructure of $\mathrm{Ni}$ is likely slightly different in the two cases. Finally, it is also possible that some of these observed intensities arise from highly reactive sp-sites at the surface region or from $\mathrm{NiC}_{x}$. Nevertheless, as stated in our earlier work, ${ }^{14}$ the metals are likely to play an important role in the electrocatalytic performance of the carbon nanomaterials and should be carefully considered when determining the root cause for their observed performance.

Based on the literature, nitrogen functionalities in a carbon matrix are often observed in graphitic, pyridinic, pyrrolic, and nitrilic forms. ${ }^{35,72,88,106,120-122}$ In contrast to photoelectron spectroscopy, $\mathrm{N}$ 1s XAS has been shown to provide relatively well defined and energetically separated peaks that represent the main nitrogen functionalities. We have aligned our dataset such that the second-order resonance of Ni $2 p$ L3 (which is available as a reference in our scans) is fixed at $852.7 \mathrm{eV}$ (426.35 eV in second order). For the comparison with other datasets, we applied further slight energy shifts to our dataset $(+0.44 \mathrm{eV})$ to match Schiros et al. peak attribution energies. Furthermore, the following shifts were applied to other reference datasets to align them with ours (after ours had been aligned with Schiros et al.): Shimoyama et al. ${ }^{122}(+0.22$ $\mathrm{eV}$ ) and Hellgren et al. ${ }^{121}(+0.17 \mathrm{eV})$ (see Figure S4 for a detailed comparison of reference spectra to our work). Our findings also match well with the studies by Zhang et al., Che et al., Zhu et al. and Dennis et al. ${ }^{35,88,106,120}$

We have indicated the most likely assignment of the main features in the dataset in Figure 1 as follows (represented by helplines): pyridinic features at $398.4 \pm 0.4 \mathrm{eV}$, nitrilic features at $399.7 \pm 0.4 \mathrm{eV}$, and graphitic features at $401.1 \pm 0.4 \mathrm{eV}$. In the sigma region (above $\sim 406 \mathrm{eV}$ ), we observe a sharp peak in graphene ${ }_{\mathrm{CVD}}$ associated with $\sigma$ for matrixed $\mathrm{N}$ in graphitic-type bonding. ${ }^{72}$ At higher energies, we observe $\sigma$ resonance associated with shorter $\mathrm{C}-\mathrm{N}$ bonds that grow in strength as more pyridinic and nitrilic features are present (see, e.g., bondlength with a ruler, Stohr).

To further investigate the chemistry and structure of the immediate surface of the carbon nanomaterials and to compare these results with the signals originating deeper from the carbon matrix, we show the Auger electron yield (AEY, $\sim 2 \mathrm{~nm}$ probing depth $\left.{ }^{123}\right)$ spectra overlayed with the TEY $(\sim 7 \mathrm{~nm}$ depth) spectra in Figure 5. As can be seen from Figure 5, spectral features (as in peak positions and relative intensities of peaks compared to the $\mathrm{sp}^{2} \pi^{*}$ peaks intensity) of all carbon nanomaterials, except for the $\mathrm{CNF}$, have strong similarities to the TEY signal. However, significant differences are observed in all of the CNF samples. The AEY spectra of all of the CNF samples can be associated with a poorly defined mixture of carbon functionalities dominated by carboxyl-like groups. All spectra also display a reduction in $\pi^{*}$ associated with $\mathrm{sp}^{2}$ type

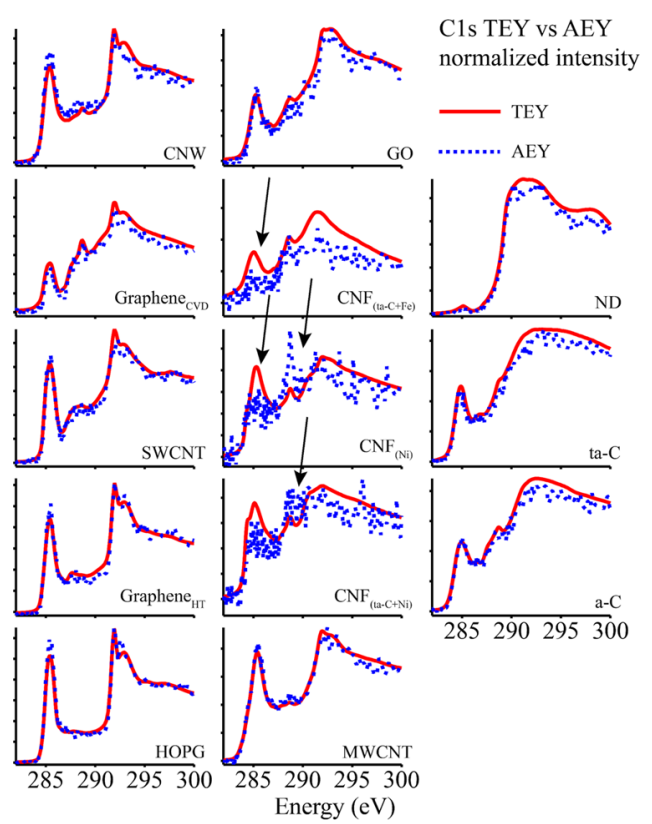

Figure 5. Total electron yield (TEY, red solid line) and Auger electron yield (AEY, dashed blue line) C 1s XAS of twelve different carbon nanomaterials accompanied by highly ordered pyrolytic graphite (HOPG). Key for abbreviations: single-wall carbon nanotube (SWCNT), carbon nanowall (CNW), multi-wall carbon nanotube (MWCNT), carbon nanofiber (CNF), graphene oxide (GO), and amorphous carbon (a-C), tetrahedral amorphous carbon (ta-C), and nanodiamond (ND). Black arrows are used to point regions of interest (clear differences between the TEY and AEY spectra).

carbon. The absolute intensity of the AEY spectra of CNF also shows lower intensity compared to other samples (see Figure S6 in the Supporting Information). This reduced amount of carbon in the surface region is likely originating from the excessive amount of metal (nanoparticles located at the tips of the fibers (see refs 58, 82, and 124 and Figure S7 in the Supporting Information)) in comparison to other analyzed carbon nanomaterials. This would also explain the higher overall amount of oxygen observed in CNFs in comparison to other carbonaceous nanomaterials investigated here since the metallic nanoparticles at the tips of the fibers have been found to be partly oxidized. ${ }^{58,82,124}$

The $\pi$ transition region of the $\mathrm{O} 1 \mathrm{~s}$ spectra is shown magnified in Figure 6. The results of the $\mathrm{O}$ 1s spectra mainly support the trends observed in the $\mathrm{C} 1 \mathrm{~s}$ region, where carboxyl-like functional groups are most abundant in the different CNM. However, there are some cases where the global C 1 s fit does not seem to completely agree with the $\mathrm{O} 1 \mathrm{~s}$ fit, for example, in the case of the clearly visible intensity of $\mathrm{H}_{2} \mathrm{C}=\mathrm{O} /$ aldehyde in CNW. Based on the $\mathrm{O}$ 1s spectra, the only materials with significant amounts of functional groups other than the higher energy ones (carboxyl and $\mathrm{C}-\mathrm{OH}$ ) are SWCNT, MWCNT, all CNF, a-C, and ta-C. Additionally, for the MWCNT, it is not clear, based on the $\mathrm{O} 1 \mathrm{~s}$ spectra, whether the increase in the $\mathrm{H}_{2} \mathrm{C}=\mathrm{O}$ /aldehyde intensity is caused by oxygen or can rather be induced by the broadening of the $\operatorname{sp}^{2} \pi^{*}$ feature.

The amount of oxygen loading on the surface of the carbon nanomaterials varies greatly. Although XAS cannot be used for quantitative analysis of the oxygen concentration, it is evidently based on the intensity of the spectra that there are differences in the amount of oxygen at the surface regions. Based on these, 

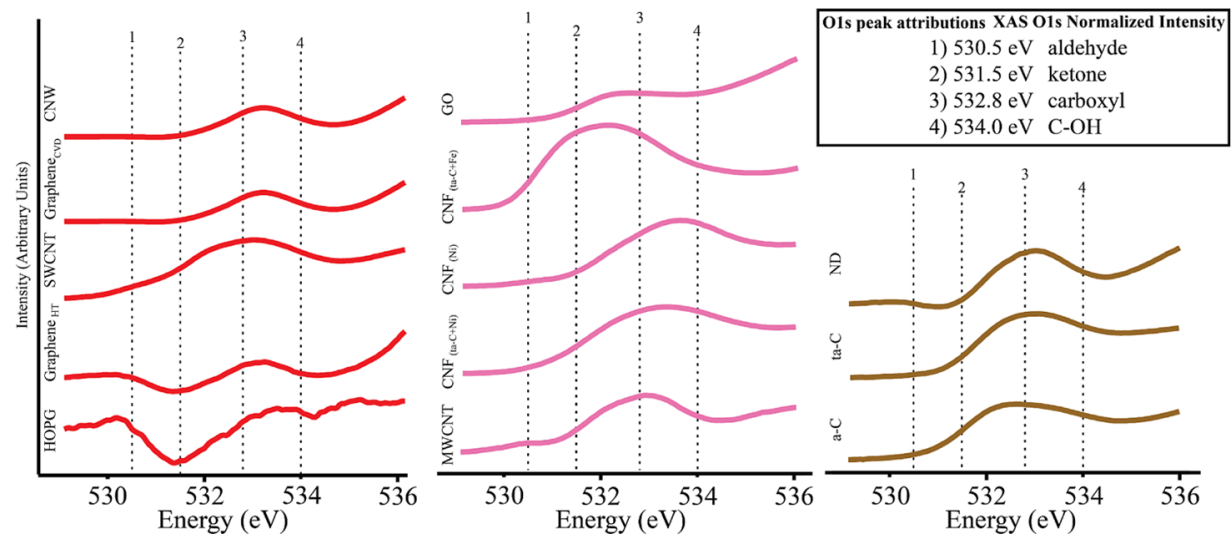

Figure 6. O 1s normalized spectra magnified and sorted into three subgroups following the division of crystalline (red), mixed (pink), and amorphous (brown-green) materials. Peak positions are marked with dashed lines to guide the eye.

Table 3. Summary of the Properties of the Studied Carbon Nanomaterials Where Their Crystallinity, C 1s Dominating Functional Group, $\mathrm{O}$ 1s Absolute Intensity, and N 1s Absolute Intensities Are Listed

\begin{tabular}{|c|c|c|c|c|c|}
\hline $\begin{array}{l}\text { property } \\
\text { summary }\end{array}$ & $\begin{array}{l}\text { carbon matrix crystallinity (long- } \\
\text { range order) }\end{array}$ & $\begin{array}{l}\text { C 1s most abundant } \\
\text { functional group }\end{array}$ & $\begin{array}{l}\text { O 1s absolute } \\
\text { intensity level }\end{array}$ & $\begin{array}{l}\mathrm{N} 1 \mathrm{~s} \text { absolute } \\
\text { intensity level }\end{array}$ & $\begin{array}{c}\text { difference between AKY } \\
\text { and TEY }\end{array}$ \\
\hline HOPG & crystalline & $\mathrm{N} / \mathrm{A}$ & not detected & not detected & no \\
\hline graphene $_{\mathrm{HT}}$ & crystalline & $\mathrm{C}-\mathrm{H}$ & low & not detected & no \\
\hline CNW & crystalline & carboxyl & low & not detected & no \\
\hline SWCNT & crystalline & $\mathrm{C}-\mathrm{H} /$ carboxyl & low & low & no \\
\hline graphene $_{\mathrm{CVD}}$ & crystalline & carboxyl & medium & low & no \\
\hline MWCNT & mixed & $\mathrm{H}_{2} \mathrm{C}=\mathrm{O} /$ aldehyde & high & not detected & no \\
\hline $\mathrm{CNF}_{(\mathrm{ta}-\mathrm{C}+\mathrm{Ni})}$ & mixed & $\mathrm{H}_{2} \mathrm{C}=\mathrm{O} /$ aldehyde & medium & medium & yes \\
\hline $\mathrm{CNF}_{(\mathrm{Ni})}$ & mixed & carboxyl & low & medium & yes \\
\hline $\mathrm{CNF}_{(\mathrm{ta}-\mathrm{C}+\mathrm{Fe})}$ & mixed & carboxyl & high & high & yes \\
\hline GO & mixed & carboxyl & medium & low & no \\
\hline $\mathrm{a}-\mathrm{C}$ & amorphous & carboxyl & low & low & no \\
\hline ta-C & amorphous & carboxyl & low & low & no \\
\hline DND & amorphous & $\mathrm{C}-\mathrm{OH}$ & low & medium & no \\
\hline
\end{tabular}

the spectra can be categorized into surfaces with (i) low concentration (graphene HT $_{1}, \mathrm{CNW}$, SWCNT, MWCNT, $\mathrm{CNF}_{(\mathrm{Ni})}$, a-C, ta-C, and $\mathrm{ND}$ ); (ii) medium concentration (graphene $_{\mathrm{CVD}}, \mathrm{CNF}_{(\mathrm{ta}-\mathrm{C}+\mathrm{Ni})}$, and $\mathrm{GO}$ ); and then (iii) high concentration $\left(\mathrm{CNF}_{(\mathrm{ta}-\mathrm{C}+\mathrm{Fe})}\right)$. It should be mentioned here that the $\mathrm{O} 1 \mathrm{~s}$ spectra work as an excellent verification source for any findings in the $\mathrm{C} 1 \mathrm{~s}$ region. The features that are truly from oxygen in the $\mathrm{C} 1 \mathrm{~s}$ spectra must also be present in the $\mathrm{O}$ 1s spectra; otherwise, it would be an indication that they are not originating from oxygen but from something else. Additionally, it is of importance to note that due the complex nature of reactions taking place at the carbon nanomaterial surface, it is recommended to study the oxygen and nitrogen edges concurrently with the carbon edge to try to understand the surface chemistry of carbon nanomaterials. Although this appears to be a trivial statement, it is not always followed in practice. This can be attributed at least partly to that in X-ray photoelectron spectroscopy (XPS), and there are significant differences in chemical shift between the different edges, i.e., $\mathrm{C}$ $1 \mathrm{~s}$ carries much more information in terms of the chemical shift between the functional groups than $\mathrm{O} 1 \mathrm{~s}$ and $\mathrm{N} 1 \mathrm{~s}$. This makes it difficult to make direct connections between the observations in $\mathrm{C} 1 \mathrm{~s}$ and $\mathrm{O} 1 \mathrm{~s} / \mathrm{N}$ 1s spectra. In XAS, there is more detailed chemical information available and due to the more detailed information available from $\mathrm{O} 1 \mathrm{~s}$ and $\mathrm{N} 1 \mathrm{~s}$ regions, the observations from $\mathrm{C}$ 1s spectra should be supported by the $\mathrm{O} 1 \mathrm{~s}$ and $\mathrm{N} 1 \mathrm{~s}$ data like that performed in this work and others.

All of the carbon nanomaterials in this study have very low amounts of nitrogen on their surfaces, as the absolute intensities of the $\mathrm{N}$ 1s spectra from all of the samples have very low counts. The only clear peak-like features are observed (from the highest to lowest counts) for ta-C + Fe CNF, ta-C + Ni CNF, DND, and Ni CNF. These materials have the highest amount of nitrogen in our dataset. The absolute intensity reveals a flat line for the rest of the materials. We can extract some more information with normalized intensity since the normalized signal reveals clear peak-shapes for SWCNT, graphene, GO, a-C, and ta-C (not in any particular order), which have medium amounts of nitrogen present. The rest of the materials, namely, MWCNT, CNW, and graphene ${ }_{\mathrm{HT}}$, have spectra nearly identical to HOPG, which is expected to be nitrogen free as discussed earlier; we expect HOPG to be a prototypical representation of pure $\mathrm{sp}^{2}$ carbon. Thus, the last group is expected to have an insignificant amount of nitrogen present. Interestingly, the $\mathrm{N}$ 1s spectra show clear differences in the types of carbon-nitrogen bonds present in the materials. The origin of these features is discussed in some detail in the literature. However, due to a large amount of different materials in our study and lack of computational resources to build models for solid carbon-nitrogen surfaces, it is not feasible to draw strong conclusions of what exact carbon- 
nitrogen groups are on the material surface. The main trends observed in the present work are summarized in Table 3.

\section{CONCLUSIONS}

In this work, we have investigated several structurally diverse carbon nanomaterials and shown clear trends in their $\mathrm{C} 1 \mathrm{~s}, \mathrm{O}$ $1 \mathrm{~s}$, and $\mathrm{N}$ 1s XAS spectra as a function of their structural and chemical properties. Based on the observed trends, (i) there is a clear correlation between the position and shape of the $\mathrm{sp}^{2}$ $\pi^{*}$ feature in the spectra, the presence of excitonic feature at $291.7 \mathrm{eV}$, and the crystallinity of the material, (ii) that carbon nanomaterials have widely different oxygen and nitrogen surface loadings, (iii) surfaces with highest oxygen concentration are the ones with no clear long-range order, (iv) nitrogen loading on the surface is more diverse than that of oxygen, and the materials dominant nitrogen group varies greatly, and (v) the amount of nitrogen on the material surface is lower than that of oxygen. Additionally, (vi) only the presence of carboxyl groups in the C 1s spectra is clearly identified as a peak, whereas the presence of other groups has to be extracted through data fitting. Importantly, (vii) the presence of carbon-oxygen bonds in $\mathrm{C}$ 1s spectra was further confirmed by inspection of the $\mathrm{O} 1 \mathrm{~s}$ spectra, which should be a general practice for the verification of the results. We also exemplify how computational studies can help to understand various previously unexplained features of the spectra, such as low energy features in $\mathrm{C} 1 \mathrm{~s}$ spectra of a-C and ta- $\mathrm{C}$ materials. Finally, this work also provides a systematic method of energy calibrating different $\mathrm{C} 1 \mathrm{~s}$ spectra to compare them using an $\mathrm{sp}^{2}$ long-range order at $291.65 \mathrm{eV}$ as a calibration point. If the $\mathrm{sp}^{2}$ long-range order is not available, then the carboxyl functional group can be used. Thus, this study serves as a reference for the chemical state and trends of carbon nanomaterials, providing an overview of the features of crystalline, mixed, and amorphous phases via XAS. Especially, our results will provide guidelines for the practitioners working with nonideal carbon nanomaterials in various fields, providing them with the previously missing map of the properties for a wide variety of nanocarbon materials. This will, for example, greatly enhance our ability to explore the connections between the electrochemical performance and surface chemistry as well as provide us with a solid basis to create realistic computational models that can further be used to deconvolute and rationalize the experimental data.

\section{ASSOCIATED CONTENT}

\section{(s) Supporting Information}

The Supporting Information is available free of charge at https://pubs.acs.org/doi/10.1021/acs.jpcc.0c08597.

Cross-correlation of results with XPS; reference spectra for diamond; Raman studies of graphene $\mathrm{CVD}_{\mathrm{C}}$; N 1s XAS spectra collection from literature; C 1 s absolute intensity comparison of AEY spectra from different samples; transmission electron microscopy (TEM) micrographs of ta-C + Ni CNF; i0 spectra from SSRL beamline 8-2; and peak fitting examples from this work (PDF)

\section{AUTHOR INFORMATION}

\section{Corresponding Author}

Sami Sainio - Microelectronics Research Unit, Faculty of Information Technology and Electrical Engineering, University of Oulu, 90570 Oulu, Finland; Stanford
Synchrotron Radiation Lightsource, SLAC National Accelerator Laboratory, Menlo Park, California 94025, United States; 10 orcid.org/0000-0002-9268-0124; Email: ssainio@slac.stanford.edu,sami.sainio@carbon.fi

\section{Authors}

Niklas Wester - Department of Chemistry and Materials Science, Aalto University, 02150 Espoo, Finland; (1) orcid.org/0000-0002-7937-9011

Anja Aarva - Department of Electrical Engineering and Automation, School of Electrical Engineering, Aalto University, 00076 Aalto, Finland

Charles J. Titus - Department of Physics, Stanford University, Stanford, California 94305, United States; (1) orcid.org/ 0000-0001-6312-8552

Dennis Nordlund - Stanford Synchrotron Radiation Lightsource, SLAC National Accelerator Laboratory, Menlo Park, California 94025, United States

Esko I. Kauppinen - Department of Applied Physics, School of Science, Aalto University, FI 00076 Aalto, Finland; (1) orcid.org/0000-0003-1727-8810

Elli Leppänen - Department of Electrical Engineering and Automation, School of Electrical Engineering, Aalto University, 00076 Aalto, Finland

Tommi Palomäki - Department of Chemistry and Materials Science, Aalto University, 02150 Espoo, Finland

Jessica E. Koehne - Center for Nanotechnology, NASA Ames Research Center, Moffett Field, California 94035, United States

Olli Pitkänen - Microelectronics Research Unit, Faculty of Information Technology and Electrical Engineering, University of Oulu, 90570 Oulu, Finland; ○ orcid.org/ 0000-0003-2870-3229

Krisztian Kordas - Microelectronics Research Unit, Faculty of Information Technology and Electrical Engineering, University of Oulu, 90570 Oulu, Finland; orcid.org/ 0000-0002-7331-1278

Maria Kim - Department of Electronics and Nanoengineering, School of Electrical Engineering, Aalto University, 00076 Aalto, Finland; Ioffe Physicotechnical Institute, Russian Academy of Sciences, St. Petersburg 194021, Russia

Harri Lipsanen - Department of Electronics and Nanoengineering, School of Electrical Engineering, Aalto University, 00076 Aalto, Finland; 이이.org/0000-00032487-4645

Miran Mozetic - Jozef Stefan Institute, 1000 Ljubljana, Slovenia

Miguel A. Caro - Department of Electrical Engineering and Automation, School of Electrical Engineering, Aalto University, 00076 Aalto, Finland; 10 orcid.org/0000-00019304-4261

M. Meyyappan - Center for Nanotechnology, NASA Ames Research Center, Moffett Field, California 94035, United States

Jari Koskinen - Department of Chemistry and Materials Science, Aalto University, 02150 Espoo, Finland

Tomi Laurila - Department of Electrical Engineering and Automation, School of Electrical Engineering, Aalto University, 00076 Aalto, Finland; (1) orcid.org/0000-0002$1252-8764$

Complete contact information is available at: https://pubs.acs.org/10.1021/acs.jpcc.0c08597 


\section{Notes}

The authors declare no competing financial interest.

\section{ACKNOWLEDGMENTS}

Carbodeon is acknowledged for providing the DND samples. Use of the Stanford Synchrotron Radiation Lightsource, SLAC National Accelerator Laboratory, is supported by the U.S. Department of Energy, Office of Science, Office of Basic Energy Sciences under contract no. DE-AC02-76SF00515. The authors wish to acknowledge CSC-IT Center for Science, Finland, for computational resources. S.S. acknowledges funding from the Instrumentarium Science Foundation and Walter Ahlström Foundation. M.Mo. acknowledges support from the Slovenian Research Agency, grant P2-0082. This project has received funding from the European Union's Horizon 2020 research and innovation program under the Marie Skłodowska-Curie grant agreement no. 841621. M.K., H.L., and K.K. acknowledge funding from the Academy of Finland, grants 298297 and 325185 (Nigella). T.L. acknowledges funding from the European Union's Horizon 2020 research and innovation program H2020-FETPROACT-201801 under grant agreement no. 824070. N.W. acknowledges funding from Emil Aaltonen Foundation and Foundation for Aalto University Science and Technology. Jarkko Etula is acknowledged for help with depositing catalyst layers for CNF growth. Henrika Granbohm is acknowledged for the synthesis of GO.

\section{REFERENCES}

(1) Han, J.-W.; Kim, B.; Li, J.; Meyyappan, M. Carbon Nanotube Based Humidity Sensor on Cellulose Paper. J. Phys. Chem. C 2012, 116, 22094-22097.

(2) Llobet, E. Gas Sensors Using Carbon Nanomaterials: A Review. Sens. Actuators, B 2013, 179, 32-45.

(3) Li, X.; Zhang, R.; Yu, W.; Wang, K.; Wei, J.; Wu, D.; Cao, A.; Li, Z.; Cheng, Y.; Zheng, Q.; et al. Stretchable and Highly Sensitive Graphene-on-Polymer Strain Sensors. Sci. Rep. 2012, 2, No. 870.

(4) Shi, J.; Li, X.; Cheng, H.; Liu, Z.; Zhao, L.; Yang, T.; Dai, Z.; Cheng, Z.; Shi, E.; Yang, L.; et al. Graphene Reinforced Carbon Nanotube Networks for Wearable Strain Sensors. Adv. Funct. Mater. 2016, 26, 2078-2084.

(5) Du, J.; Pei, S.; Ma, L.; Cheng, H.-M. 25th Anniversary Article: Carbon Nanotube- and Graphene-Based Transparent Conductive Films for Optoelectronic Devices. Adv. Mater. 2014, 26, 1958-1991.

(6) Atkinson, K.; Roth, S.; Hirscher, M.; Grünwald, W. Carbon Nanostructures: An Efficient Hydrogen Storage Medium for Fuel Cells. Fuel Cells Bull. 2001, 4, 9-12.

(7) Zhu, J.; Holmen, A.; Chen, D. Carbon Nanomaterials in Catalysis: Proton Affinity, Chemical and Electronic Properties, and Their Catalytic Consequences. Chem CatChem 2013, 5, 378-401.

(8) Hu, Y.; Shenderova, O. A.; Hu, Z.; Padgett, C. W.; Brenner, D. W. Carbon Nanostructures for Advanced Composites. Rep. Prog. Phys. 2006, 69, 1847.

(9) Mendes, R. G.; Bachmatiuk, A.; Büchner, B.; Cuniberti, G.; Rümmeli, M. H. Carbon Nanostructures as Multi-Functional Drug Delivery Platforms. J. Mater. Chem. B 2013, 1, 401-428.

(10) Robertson, J. Diamond-like Amorphous Carbon. Mater. Sci. Eng., $R$ 2002, 37, 129-281.

(11) Anisimov, A. S.; Brown, D. P.; Mikladal, B. F.; Súilleabháin, L. Ó; Parikh, K.; Soininen, E.; Sonninen, M.; Tian, D.; Varjos, I.; Vuohelainen, R. 16.3: Printed Touch Sensors Using Carbon NanoBud Material. SID Symp. Dig. Tech. Pap. 2014, 45, 200-203.

(12) Zhu, Y.; Ji, H.; Cheng, H.-M.; Ruoff, R. S. Mass Production and Industrial Applications of Graphene Materials. Natl. Sci. Rev. 2018, 5, 90-101.
(13) Zhang, Q.; Huang, J.-Q.; Qian, W.-Z.; Zhang, Y.-Y.; Wei, F. The Road for Nanomaterials Industry: A Review of Carbon Nanotube Production, Post-Treatment, and Bulk Applications for Composites and Energy Storage. Small 2013, 9, 1237-1265.

(14) Sainio, S.; Leppänen, E.; Mynttinen, E.; Palomäki, T.; Wester, N.; Etula, J.; Isoaho, N.; Peltola, E.; Koehne, J.; Meyyappan, M.; et al. Integrating Carbon Nanomaterials with Metals for Bio-Sensing Applications. Mol. Neurobiol. 2020, 57, 179-190.

(15) Laurila, T.; Rautiainen, A.; Sintonen, S.; Jiang, H.; Kaivosoja, E.; Koskinen, J. Diamond-like Carbon (DLC) Thin Film Bioelectrodes: Effect of Thermal Post-Treatments and the Use of Ti Adhesion Layer. Mater. Sci. Eng., C 2014, 34, 446-454.

(16) Isoaho, N.; Peltola, E.; Sainio, S.; Koskinen, J.; Laurila, T. PtGrown Carbon Nanofibers for Enzymatic Glutamate Biosensors and Assessment of Their Biocompatibility. RSC Adv. 2018, 8, 3580235812 .

(17) Peltola, E.; Wester, N.; Holt, K. B.; Johansson, L.-S.; Koskinen, J.; Myllymäki, V.; Laurila, T. Nanodiamonds on Tetrahedral Amorphous Carbon Significantly Enhance Dopamine Detection and Cell Viability. Biosens. Bioelectron. 2017, 88, 273-282.

(18) Elomaa, O.; Singh, V. K.; Iyer, A.; Hakala, T. J.; Koskinen, J. Graphene Oxide in Water Lubrication on Diamond-like Carbon vs. Stainless Steel High-Load Contacts. Diamond Relat. Mater. 2015, 52, $43-48$.

(19) Laurila, T.; Sainio, S.; Caro, M. A. Hybrid Carbon Based Nanomaterials for Electrochemical Detection of Biomolecules. Prog. Mater. Sci. 2017, 88, 499-594.

(20) Watts, B.; Swaraj, S.; Nordlund, D.; Lüning, J.; Ade, H. Calibrated NEXAFS Spectra of Common Conjugated Polymers. J. Chem. Phys. 2011, 134, No. 024702.

(21) Urquhart, S. G.; Ade, H. Trends in the Carbonyl Core (C 1S, O 1S) $\rightarrow \pi^{*} \mathrm{C}=\mathrm{O}$ Transition in the Near-Edge $\mathrm{X}$-Ray Absorption Fine Structure Spectra of Organic Molecules. J. Phys. Chem. B 2002, 106, $8531-8538$.

(22) Bergmann, U.; Mullins, O. C. Carbon X-ray Raman Spectroscopy of PAHs and Asphaltenes. In Asphaltenes, Heavy Oils, and Petroleomics; Mullins, O. C.; Sheu, E. Y.; Hammami, A.; Marshall, A. G., Eds.; Springer: New York, NY, 2007; pp 139-155.

(23) Pomerantz, A. E.; Crace, E.; Weng, T.-C.; Sokaras, D.; Nordlund, D. Carbon Core Electron Spectra of Polycyclic Aromatic Hydrocarbons. J. Phys. Chem. A 2018, 122, 5730-5734.

(24) Lehmann, J.; Liang, B.; Solomon, D.; Lerotic, M.; Luizão, F.; Kinyangi, J.; Schäfer, T.; Wirick, S.; Jacobsen, C. Near-Edge X-Ray Absorption Fine Structure (NEXAFS) Spectroscopy for Mapping Nano-Scale Distribution of Organic Carbon Forms in Soil: Application to Black Carbon Particles. Global Biogeochem. Cycles 2005, 19, 21.

(25) Solomon, D.; Lehmann, J.; Kinyangi, J.; Liang, B.; Schäfer, T. Carbon K-Edge NEXAFS and FTIR-ATR Spectroscopic Investigation of Organic Carbon Speciation in Soils. Soil Sci. Soc. Am. J. 2005, 69, 107.

(26) Lehmann, J.; Solomon, D. Organic Carbon Chemistry in Soils Observed by Synchrotron-Based Spectroscopy. In Developments in Soil Science; Singh, B.; Gräfe, M., Eds.; Elsevier, 2010; Chapter 10, Vol. 34, pp 289-312.

(27) Solomon, D.; Lehmann, J.; Kinyangi, J.; Liang, B.; Heymann, K.; Dathe, L.; Hanley, K.; Wirick, S.; Jacobsen, C. Carbon (1s) NEXAFS Spectroscopy of Biogeochemically Relevant Reference Organic Compounds. Soil Sci. Soc. Am. J. 2009, 73, 1817-1830.

(28) Hitchcock, A. P. Core Excitation and Ionization of Molecules. Phys. Scr. 1990, 1990, 159.

(29) Ishii, I.; Hitchcook, A. P. The Oscillator Strengths for C1s and O1s Excitation of Some Saturated and Unsaturated Organic Alcohols, Acids and Esters. J. Electron Spectrosc. Relat. Phenom. 1988, 46, 55-84.

(30) Urquhart, S. G.; Hitchcock, A. P.; Priester, R. D.; Rightor, E. G. Analysis of Polyurethanes Using Core Excitation Spectroscopy. Part II: Inner Shell Spectra of Ether, Urea and Carbamate Model Compounds. J. Polym. Sci., Part B: Polym. Phys. 1995, 1603-1620. 
(31) Zhao, L.; He, R.; Rim, K. T.; Schiros, T.; Kim, K. S.; Zhou, H.; Gutiérrez, C.; Chockalingam, S. P.; Arguello, C. J.; Pálová, L.; et al. Visualizing Individual Nitrogen Dopants in Monolayer Graphene. Science 2011, 333, 999-1003.

(32) Rossouw, D.; Botton, G. A.; Najafi, E.; Lee, V.; Hitchcock, A. P. Metallic and Semiconducting Single-Walled Carbon Nanotubes: Differentiating Individual SWCNTs by Their Carbon 1s Spectra. ACS Nano 2012, 6, 10965-10972.

(33) Kuznetsova, A.; Popova, I.; Yates, J. T., Jr.; Bronikowski, M. J.; Huffman, C. B.; Liu, J.; Smalley, R. E.; Hwu, H. H.; Chen, J. G. Oxygen-Containing Functional Groups on Single-Wall Carbon Nanotubes: NEXAFS and Vibrational Spectroscopic Studies. J. Am. Chem. Soc. 2001, 123, 10699-10704.

(34) Mowbray, D. J.; Ayala, P.; Pichler, T.; Rubio, A. Computing C1s X-Ray Absorption for Single-Walled Carbon Nanotubes with Distinct Electronic Type. Mater. Express 2011, 1, 225-230.

(35) Zhang, J.-R.; Ma, Y.; Wang, S.-Y.; Ding, J.; Gao, B.; Kan, E.; Hua, W. Accurate K-Edge X-Ray Photoelectron and Absorption Spectra of G-C3N4 Nanosheets by First-Principles Simulations and Reinterpretations. Phys. Chem. Chem. Phys. 2019, 21, 22819-22830.

(36) Terminello, L. J.; Shuh, D. K.; Himpsel, F. J.; Lapiano-Smith, D. A.; Stöhr, J.; Bethune, D. S.; Meijer, G. Unfilled Orbitals of C60 and C70 from Carbon K-Shell X-Ray Absorption Fine Structure. Chem. Phys. Lett. 1991, 182, 491-496.

(37) Hoffman, A.; Laikhtman, A. Photon Stimulated Desorption of Hydrogen from Diamond Surfaces via Core-Level Excitations: Fundamental Processes and Applications to Surface Studies. J. Phys.: Condens. Matter 2006, 18, S1517.

(38) Zagrebina, E. M.; Generalov, A. V.; Klyushin, A. Y.; Simonov, K. A.; Vinogradov, N. A.; Dubois, M.; Frezet, L.; Mårtensson, N.; Preobrajenski, A. B.; Vinogradov, A. S. Comparative NEXAFS, NMR, and FTIR Study of Various-Sized Nanodiamonds: As-Prepared and Fluorinated. J. Phys. Chem. C 2015, 119, 835-844.

(39) Ziethen, C.; Schmidt, O.; Marx, G. K. L.; Schönhense, G.; Frömter, R.; Gilles, J.; Kirschner, J.; Schneider, C. M.; Gröning, O. Orbital Mapping of Carbon Thin Films by XANES-Spectromicroscopy. J. Electron Spectrosc. Relat. Phenom. 2000, 107, 261-271.

(40) Vi, K.; Sladkov, A. M.; Yp, K.; Popov, N. M.; Korshak, V. V. Crystalline Forms of Linear Modification of Carbon. Dokl. Akad. Nauk SSSR 1967, 177, 358.

(41) Shi, L.; Rohringer, P.; Suenaga, K.; Niimi, Y.; Kotakoski, J.; Meyer, J. C.; Peterlik, H.; Wanko, M.; Cahangirov, S.; Rubio, A.; et al. Confined Linear Carbon Chains as a Route to Bulk Carbyne. Nat. Mater. 2016, 15, 634-639.

(42) Liu, M.; Artyukhov, V. I.; Lee, H.; Xu, F.; Yakobson, B. I. Carbyne from First Principles: Chain of $\mathrm{C}$ Atoms, a Nanorod or a Nanorope. ACS Nano 2013, 7, 10075-10082.

(43) Banks, C. E.; Moore, R. R.; Davies, T. J.; Compton, R. G. Investigation of Modified Basal Plane Pyrolytic Graphite Electrodes: Definitive Evidence for the Electrocatalytic Properties of the Ends of Carbon Nanotubes. Chem. Commun. 2004, 16, 1804-1805.

(44) Banks, C. E.; Davies, T. J.; Wildgoose, G. G.; Compton, R. G. Electrocatalysis at Graphite and Carbon Nanotube Modified Electrodes: Edge-Plane Sites and Tube Ends Are the Reactive Sites. Chem. Commun. 2005, 829-841.

(45) Sainio, S.; Nordlund, D.; Caro, M. A.; Gandhiraman, R.; Koehne, J.; Wester, N.; Koskinen, J.; Meyyappan, M.; Laurila, T. Correlation between sp3-to-sp2 Ratio and Surface Oxygen Functionalities in Tetrahedral Amorphous Carbon (ta-C) Thin Film Electrodes and Implications of Their Electrochemical Properties. J. Phys. Chem. C 2016, 120, 8298-8304.

(46) Wester, N.; Sainio, S.; Palomäki, T.; Nordlund, D.; Singh, V. K.; Johansson, L.-S.; Koskinen, J.; Laurila, T. Partially Reduced Graphene Oxide Modified Tetrahedral Amorphous Carbon ThinFilm Electrodes as a Platform for Nanomolar Detection of Dopamine. J. Phys. Chem. C 2017, 121, 8153-8164.

(47) Peltola, E.; Heikkinen, J. J.; Sovanto, K.; Sainio, S.; Aarva, A.; Franssila, S.; Jokinen, V.; Laurila, T. SU-8 Based Pyrolytic Carbon for the Electrochemical Detection of Dopamine. J. Mater. Chem. B 2017, 5, 9033-9044.

(48) Wang, L.; Sofer, Z.; Pumera, M. Will Any Crap We Put into Graphene Increase Its Electrocatalytic Effect? ACS Nano 2020, 14, 21-25.

(49) Novoselov, K. S.; Geim, A. K.; Morozov, S. V.; Dubonos, S. V.; Zhang, Y.; Jiang, D. Room-Temperature Electric Field Effect and Carrier-Type Inversion in Graphene Films. 2004, arXiv:/cond-mat/ 0410631. arXiv.org e-Print archive. https://arxiv.org/abs/cond-mat/ 0410631.

(50) Kovtyukhova, N. I.; Ollivier, P. J.; Martin, B. R.; Mallouk, T. E.; Chizhik, S. A.; Buzaneva, E. V.; Gorchinskiy, A. D. Layer-by-Layer Assembly of Ultrathin Composite Films from Micron-Sized Graphite Oxide Sheets and Polycations. Chem. Mater. 1999, 11, 771-778.

(51) Hummers, W. S.; Offeman, R. E. Preparation of Graphitic Oxide. J. Am. Chem. Soc. 1958, 80, 1339.

(52) Granbohm, H.; Kulmala, K.; Iyer, A.; Ge, Y.; Hannula, S.-P. Preparation and Photocatalytic Activity of Quaternary GO/TiO2/Ag/ $\mathrm{AgCl}$ Nanocomposites. Water, Air, Soil Pollut. 2017, 228, 127.

(53) Palomäki, T.; Wester, N.; Johansson, L.-S.; Laitinen, M.; Jiang, H.; Arstila, K.; Sajavaara, T.; Han, J. G.; Koskinen, J.; Laurila, T. Characterization and Electrochemical Properties of Oxygenated Amorphous Carbon (a-C) Films. Electrochim. Acta 2016, 220, 137145.

(54) Palomäki, T.; Caro, M. A.; Wester, N.; Sainio, S.; Etula, J.; Johansson, L.; Han, J. G.; Koskinen, J.; Laurila, T. Effect of Power Density on the Electrochemical Properties of Undoped Amorphous Carbon (a-C) Thin Films. Electroanalysis 2019, 31, 746-755.

(55) Novikov, S.; Lebedeva, N.; Satrapinski, A.; Walden, J. Graphene Based Sensor for Environmental Monitoring of NO2. Procedia Eng. 2015, 120, 586-589.

(56) Myllymäki, V. Zeta Positive Hydrogenated Nanodiamond Powder, Zeta Positive Single Digit Hydrogenated Nanodiamond Dispersion, and Methods for Producing The Same. US9,884,767, Dec 4, 2014.

(57) Palomäki, T.; Wester, N.; Caro, M. A.; Sainio, S.; Protopopova, V.; Koskinen, J.; Laurila, T. Electron Transport Determines the Electrochemical Properties of Tetrahedral Amorphous Carbon (ta-C) Thin Films. Electrochim. Acta 2017, 225, 1-10.

(58) Sainio, S.; Jiang, H.; Caro, M. A.; Koehne, J.; Lopez-Acevedo, O.; Koskinen, J.; Meyyappan, M.; Laurila, T. Structural Morphology of Carbon Nanofibers Grown on Different Substrates. Carbon 2016, 98, 343-351.

(59) Sainio, S.; Palomäki, T.; Rhode, S.; Kauppila, M.; Pitkänen, O.; Selkälä, T.; Toth, G.; Moram, M.; Kordas, K.; Koskinen, J.; et al. Carbon Nanotube (CNT) Forest Grown on Diamond-like Carbon (DLC) Thin Films Significantly Improves Electrochemical Sensitivity and Selectivity towards Dopamine. Sens. Actuators, B 2015, 211, 177186

(60) Pitkänen, O.; Halonen, N.; Leino, A.-R.; Mäklin, J.; Dombovári, Á.; Lin, J. H.; Tóth, G.; Kordás, K. Low-Temperature Growth of Carbon Nanotubes on Bi- and Tri-Metallic Catalyst Templates. Top. Catal. 2013, 56, 522-526.

(61) Sainio, S.; Wester, N.; Titus, C. J.; Liao, Y.; Zhang, Q.; Nordlund, D.; Sokaras, D.; Lee, S.-J.; Irwin, K. D.; Doriese.; et al. Hybrid X-Ray Spectroscopy-Based Approach To Acquire Chemical and Structural Information of Single-Walled Carbon Nanotubes with Superior Sensitivity. J. Phys. Chem. C 2019, 123, 6114-6120.

(62) Vanrenterghem, B.; Hodnik, N.; Bele, M.; Sala, M.; Amelinckx, G.; Neukermans, S.; Zaplotnik, R.; Primc, G.; Mozetič, M.; Breugelmans, T. Increase of Electrodeposited Catalyst Stability via Plasma Grown Vertically Oriented Graphene Nanoparticle Movement Restriction. Chem. Commun. 2017, 53, 9340-9343.

(63) Zaplotnik, R.; Mozetic, M.; Primc, G.; Vesel, A.; Hori, M. Carbon Nanostructured Materials and Methods for Forming Carbon Nanostructured Materials. WO2019/238206, Dec 19, 2019.

(64) Stöhr, J. NEXAFS Spectroscopy; Springer: Berlin, 2010. 
(65) Watts, B.; Thomsen, L.; Dastoor, P. C. Methods in Carbon KEdge NEXAFS: Experiment and Analysis. J. Electron Spectrosc. Relat. Phenom. 2006, 151, 105-120.

(66) Watts, B.; Ade, H. A Simple Method for Determining Linear Polarization and Energy Calibration of Focused Soft X-Ray Beams. J. Electron Spectrosc. Relat. Phenom. 2008, 162, 49-55.

(67) Brühwiler, P. A.; Maxwell, A. J.; Puglia, C.; Nilsson, A.; Andersson, S.; Mårtensson, N. $\pi^{*}$ and $\sigma^{*}$ Excitons in C $1 \mathrm{~S}$ Absorption of Graphite. Phys. Rev. Lett. 1995, 74, 614.

(68) Ma, Y.; Skytt, P.; Wassdahl, N.; Glans, P.; Guo, J.; Nordgren, J. Core Excitons and Vibronic Coupling in Diamond and Graphite. Phys. Rev. Lett. 1993, 71, 3725-3728.

(69) Aarva, A.; Deringer, V. L.; Sainio, S.; Laurila, T.; Caro, M. A. Understanding X-Ray Spectroscopy of Carbonaceous Materials by Combining Experiments, Density Functional Theory, and Machine Learning. Part I: Fingerprint Spectra. Chem. Mater. 2019, 31, 92439255.

(70) Batson, P. E. Carbon 1s near-Edge-Absorption Fine Structure in Graphite. Phys. Rev. B 1993, 48, 2608-2610.

(71) Laikhtman, A.; Gouzman, I.; Hoffman, A.; Comtet, G.; Hellner, L.; Dujardin, G. Sensitivity of near-Edge X-Ray Absorption Fine Structure Spectroscopy to Ion Beam Damage in Diamond Films. J. Appl. Phys. 1999, 86, 4192-4198.

(72) Schiros, T.; Nordlund, D.; Pálová, L.; Prezzi, D.; Zhao, L.; Kim, K. S.; Wurstbauer, U.; Gutiérrez, C.; Delongchamp, D.; Jaye, C.; et al. Connecting Dopant Bond Type with Electronic Structure in NDoped Graphene. Nano Lett. 2012, 12, 4025-4031.

(73) Skytt, P.; Glans, P.; Mancini, D. C.; Guo, J.; Wassdahl, N.; Nordgren, J.; Ma, Y. Angle-Resolved Soft-X-Ray Fluorescence and Absorption Study of Graphite. Phys. Rev. B 1994, 50, 10457-10461. (74) Brühwiler, P. A.; Maxwell, A.; Puglia, C.; Nilsson, A.; Andersson, S.; Mårtensson, N. Pi * and Sigma * Excitons in C 1s Absorption of Graphite. Phys. Rev. Lett. 1995, 74, 614-617.

(75) Enkovaara, J.; Rostgaard, C.; Mortensen, J. J.; Chen, J.; Dułak, M.; Ferrighi, L.; Gavnholt, J.; Glinsvad, C.; Haikola, V.; Hansen, H. A.; et al. Electronic Structure Calculations with GPAW: A Real-Space Implementation of the Projector Augmented-Wave Method. Phys. Rev. B 2010, No. 253202.

(76) Mortensen, J. J.; Hansen, L. B.; Jacobsen, K. W. Real-Space Grid Implementation of the Projector Augmented Wave Method. Phys. Rev. B 2005, 71, No. 035109.

(77) Perdew, J. P.; Burke, K.; Ernzerhof, M. Generalized Gradient Approximation Made Simple [Phys. Rev. Lett. 77, 3865 (1996)]. Phys. Rev. Lett. 1997, 78, 1396.

(78) Tkatchenko, A.; Scheffler, M. Accurate Molecular van Der Waals Interactions from Ground-State Electron Density and FreeAtom Reference Data. Phys. Rev. Lett. 2009, 102, No. 073005.

(79) Taillefumier, M.; Cabaret, D.; Flank, A.-M.; Mauri, F. X-Ray Absorption near-Edge Structure Calculations with the Pseudopotentials: Application to the K Edge in Diamond and \alpha-Quartz. Phys. Rev. B 2002, 66, No. 195107.

(80) Ljungberg, M. P.; Mortensen, J. J.; Pettersson, L. G. M. An Implementation of Core Level Spectroscopies in a Real Space Projector Augmented Wave Density Functional Theory Code. J. Electron Spectrosc. Relat. Phenom. 2011, 427-439.

(81) Sainio, S.; Nordlund, D.; Caro, M. A.; Gandhiraman, R.; Koehne, J.; Wester, N.; Koskinen, J.; Meyyappan, M.; Laurila, T. Correlation between sp3-to-sp2 Ratio and Surface Oxygen Functionalities in Tetrahedral Amorphous Carbon (ta-C) Thin Film Electrodes and Implications of Their Electrochemical Properties. J. Phys. Chem. C 2016, 120, 8298-8304.

(82) Sainio, S.; Nordlund, D.; Gandhiraman, R.; Jiang, H.; Koehne, J.; Koskinen, J.; Meyyappan, M.; Laurila, T. What Does Nitric Acid Really Do to Carbon Nanofibers? J. Phys. Chem. C 2016, 120, 2265522662.

(83) Larciprete, R.; Fabris, S.; Sun, T.; Lacovig, P.; Baraldi, A.; Lizzit, S. Dual Path Mechanism in the Thermal Reduction of Graphene Oxide. J. Am. Chem. Soc. 2011, 133, 17315-17321.
(84) Díaz, J.; Anders, S.; Cossy-Favre, A.; Samant, M.; Stöhr, J. Enhanced Secondary Electron Yield from Oxidized Regions on Amorphous Carbon Films Studied by X-Ray Spectromicroscopy. J. Vac. Sci. Technol., A 1999, 17, 2737-2740.

(85) Outka, D. A.; Stöhr, J.; Madix, R. J.; Rotermund, H. H.; Hermsmeier, B.; Solomon, J. NEXAFS Studies of Complex Alcohols and Carboxylic Acids on the Si(111)(7×7) Surface. Surf. Sci. 1987, $185,53-74$.

(86) Raty, J.-Y.; Galli, G.; Bostedt, C.; Van Buuren, T. W.; Terminello, L. J. Quantum Confinement and Fullerenelike Surface Reconstructions in Nanodiamonds. Phys. Rev. Lett. 2003, 90, No. 037401.

(87) Shpilman, Z.; Gouzman, I.; Minton, T. K.; Shen, L.; Stacey, A.; Orwa, J.; Prawer, S.; Cowie, B. C. C.; Hoffman, A. A near Edge X-Ray Absorption Fine Structure Study of Oxidized Single Crystal and Polycrystalline Diamond Surfaces. Diamond Relat. Mater. 2014, 45, 20-27.

(88) Dennis, R. V.; Schultz, B. J.; Jaye, C.; Wang, X.; Fischer, D. A.; Cartwright, A. N.; Banerjee, S. Near-Edge X-Ray Absorption Fine Structure Spectroscopy Study of Nitrogen Incorporation in Chemically Reduced Graphene Oxide. J. Vac. Sci. Technol., B: Nanotechnol. Microelectron.: Mater., Process., Meas., Phenom. 2013, 31, No. 041204.

(89) Lee, D. W.; De Los Santos, L. V.; Seo, J. W.; Leon Felix, L.; Bustamante, D. A.; Cole, J. M.; Barnes, C. H. W. The Structure of Graphite Oxide: Investigation of Its Surface Chemical Groups. J. Phys. Chem. B 2010, 114, 5723-5728.

(90) Mele, E. J.; Ritsko, J. J. Fermi-Level Lowering and the Core Exciton Spectrum of Intercalated Graphite. Phys. Rev. Lett. 1979, 43, $68-71$.

(91) Rosenberg, R. A.; Love, P. J.; Rehn, V. V. PolarizationDependent $\mathrm{C}(\mathrm{K})$ near-Edge X-Ray-Absorption Fine Structure of Graphite. Phys. Rev. B 1986, 33, 4034-4037.

(92) Aarva, A.; Deringer, V. L.; Sainio, S.; Laurila, T.; Caro, M. A. Understanding X-Ray Spectroscopy of Carbonaceous Materials by Combining Experiments, Density Functional Theory, and Machine Learning. Part II: Quantitative Fitting of Spectra. Chem. Mater. 2019, 31, 9256-9267.

(93) Hunt, A.; Kurmaev, E. Z.; Moewes, A. A Re-Evaluation of How Functional Groups Modify the Electronic Structure of Graphene Oxide. Adv. Mater. 2014, 26, 4870-4874.

(94) Coffman, F. L.; Cao, R.; Pianetta, P. A.; Kapoor, S.; Kelly, M.; Terminello, L. J. Near-edge X-ray Absorption of Carbon Materials for Determining Bond Hybridization in Mixed sp2/sp3 Bonded Materials. Appl. Phys. Lett. 1996, 69, 568-570.

(95) Olson, D. S.; Kelly, M. A.; Kapoor, S.; Hagstrom, S. B. Growth of Diamond from Sputtered Atomic Carbon and Atomic Hydrogen. MRS Proc. 1992, 270, No. 335.

(96) Kelly, M. A.; Olson, D. S.; Kapoor, S.; Hagstrom, S. B. Diamond Growth by a New Method Based upon Sequential Exposure to Atomic Carbon and Hydrogen. Appl. Phys. Lett. 1992, 60, 25022504.

(97) Mangolini, F.; McClimon, J. B.; Carpick, R. W. Quantitative Evaluation of the Carbon Hybridization State by Near Edge X-Ray Absorption Fine Structure Spectroscopy. Anal. Chem. 2016, 88, $2817-2824$

(98) Mangolini, F.; Brandon McClimon, J.; Rose, F.; Carpick, R. W. Accounting for Nanometer-Thick Adventitious Carbon Contamination in X-Ray Absorption Spectra of Carbon-Based Materials. Anal. Chem. 2014, 12258-12265.

(99) Sumant, A. V.; Gilbert, P. U. P. A.; Grierson, D. S.; Konicek, A. R.; Abrecht, M.; Butler, J. E.; Feygelson, T.; Rotter, S. S.; Carpick, R. W. Surface Composition, Bonding, and Morphology in the Nucleation and Growth of Ultra-Thin, High Quality Nanocrystalline Diamond Films. Diamond Relat. Mater. 2007, 16, 718-724.

(100) Gago, R.; Vinnichenko, M.; Jäger, H. U.; Belov, A. Y.; Jiménez, I.; Huang, N.; Sun, H.; Maitz, M. F. Evolution of S P 2 Networks with Substrate Temperature in Amorphous Carbon Films: Experiment and Theory. Phys. Rev. B: Condens. Matter Mater. Phys. 2005, 72, No. 014120. 
(101) Gago, R.; Jimenez, I.; Albella, J. M.; Climent-Font, A.; Caceres, D.; Vergara, I.; Banks, J. C.; Doyle, B. L.; Terminello, L. J. Bonding and Hardness in Nonhydrogenated Carbon Films with Moderate Sp 3 Content. J. Appl. Phys. 2000, 87, 8174-8180.

(102) Golze, D.; Wilhelm, J.; van Setten, M. J.; Rinke, P. Core-Level Binding Energies from GW: An Efficient Full-Frequency Approach within a Localized Basis. J. Chem. Theory Comput. 2018, 14, 48564869.

(103) Golze, D.; Dvorak, M.; Rinke, P. The GW Compendium: A Practical Guide to Theoretical Photoemission Spectroscopy. Front. Chem. 2019, 7, No. 377.

(104) Michelitsch, G. S.; Reuter, K. Efficient Simulation of nearEdge X-Ray Absorption Fine Structure (NEXAFS) in DensityFunctional Theory: Comparison of Core-Level Constraining Approaches. J. Chem. Phys. 2019, 150, No. 074104.

(105) Jiménez, I.; Tong, W. M.; Shuh, D. K.; Holloway, B. C.; Kelly, M. A.; Pianetta, P.; Terminello, L. J.; Himpsel, F. J. Bonding Modifications in Carbon Nitride Films Induced by Thermal Annealing: An X-ray Absorption near Edge Study. Appl. Phys. Lett. 1999, 74, 2620-2622.

(106) Zhu, Q.; Money, S. L.; Russell, A. E.; Thomas, K. M. Determination of the Fate of Nitrogen Functionality in Carbonaceous Materials during Pyrolysis and Combustion Using X-Ray Absorption Near Edge Structure Spectroscopy. Langmuir 1997, 13, 2149-2157. (107) Jiménez, I.; Gago, R.; Albella, J. M.; Cáceres, D.; Vergara, I. Spectroscopy of \ensuremath $\{\backslash$ pi $\}$ Bonding in Hard Graphitic Carbon Nitride Films: Superstructure of Basal Planes and Hardening Mechanisms. Phys. Rev. B 2000, 62, 4261-4264.

(108) Boese, J.; Osanna, A.; Jacobsen, C.; Kirz, J. Carbon Edge XANES Spectroscopy of Amino Acids and Peptides. J. Electron Spectrosc. Relat. Phenom. 1997, 85, 9-15.

(109) Solomon, J. L.; Madix, R. J.; Stöhr, J. Orientation and Absolute Coverage of Benzene, Aniline, and Phenol on $\mathrm{Ag}(110)$ Determined by NEXAFS and XPS. Surf. Sci. 1991, 255, 12-30.

(110) Otero, E.; Wilks, R. G.; Regier, T.; Blyth, R. I. R.; Moewes, A.; Urquhart, S. G. Substituent Effects in the Iron $2 p$ and Carbon 1s Edge near-Edge X-Ray Absorption Fine Structure (NEXAFS) Spectroscopy of Ferrocene Compounds. J. Phys. Chem. A 2008, 112, 624-634.

(111) Brühwiler, P. A. Synchrotron Studies of Carbon Surfaces. J. Phys.: Condens. Matter 2001, 13, 11229.

(112) Abbas, M.; Wu, Z. Y.; Zhong, J.; Ibrahim, K.; Fiori, A.; Orlanducci, S.; Sessa, V.; Terranova, M. L.; Davoli, I. X-Ray Absorption and Photoelectron Spectroscopy Studies on Graphite and Single-Walled Carbon Nanotubes: Oxygen Effect. Appl. Phys. Lett. 2005, 87, No. 051923.

(113) Kumar, P. V.; Bernardi, M.; Grossman, J. C. The Impact of Functionalization on the Stability, Work Function, and Photoluminescence of Reduced Graphene Oxide. ACS Nano 2013, 7, $1638-1645$

(114) Aarva, A.; Deringer, V. L.; Sainio, S.; Laurila, T.; Caro, M. A. Understanding X-Ray Spectroscopy of Carbonaceous Materials by Combining Experiments, Density Functional Theory, and Machine Learning. Part I: Fingerprint Spectra. Chem. Mater. 2019, 31, 92439255.

(115) Sainio, S.; Wester, N.; Titus, C. J.; Nordlund, D.; Lee, S.-J.; Koskinen, J.; Laurila, T. In-Situ Functionalization of Tetrahedral Amorphous Carbon by Filtered Cathodic Arc Deposition. AIP Adv. 2019, 9, No. 085325.

(116) Bergmann, U.; Groenzin, H.; Mullins, O. C.; Glatzel, P.; Fetzer, J.; Cramer, S. P. Carbon K-Edge X-Ray Raman Spectroscopy Supports Simple, yet Powerful Description of Aromatic Hydrocarbons and Asphaltenes. Chem. Phys. Lett. 2003, 369, 184-191.

(117) Gordon, M. L.; Tulumello, D.; Cooper, G.; Hitchcock, A. P.; Glatzel, P.; Mullins, O. C.; Cramer, S. P.; Bergmann, U. Inner-Shell Excitation Spectroscopy of Fused-Ring Aromatic Molecules by Electron Energy Loss and X-Ray Raman Techniques. J. Phys. Chem. A 2003, 107, 8512-8520.
(118) Schultz, B. J.; Jaye, C.; Lysaght, P. S.; Fischer, D. A.; Prendergast, D.; Banerjee, S. On Chemical Bonding and Electronic Structure of Graphene-metal Contacts. Chem. Sci. 2013, 4, 494-502.

(119) Huttmann, F.; Klar, D.; Atodiresei, N.; Schmitz-Antoniak, C.; Smekhova, A.; Martínez-Galera, A. J.; Caciuc, V.; Bihlmayer, G.; Blügel, S.; Michely, T.; et al. Magnetism in a Graphene-4 F- 3 D Hybrid System. Phys. Rev. B: Condens. Matter Mater. Phys. 2017, 95, No. 075427.

(120) Che, W.; Cheng, W.; Yao, T.; Tang, F.; Liu, W.; Su, H.; Huang, Y.; Liu, Q.; Liu, J.; Hu, F.; et al. Fast Photoelectron Transfer in (Cring)-C3N4 Plane Heterostructural Nanosheets for Overall Water Splitting. J. Am. Chem. Soc. 2017, 139, 3021-3026.

(121) Hellgren, N.; Guo, J.; Luo, Y.; Såthe, C.; Agui, A.; Kashtanov, S.; Nordgren, J.; Ågren, H.; Sundgren, J.-E. Electronic Structure of Carbon Nitride Thin Films Studied by X-ray Spectroscopy Techniques. Thin Solid Films 2005, 471, 19-34.

(122) Shimoyama, I.; Wu, G.; Sekiguchi, T.; Baba, Y. Evidence for the Existence of Nitrogen-Substituted Graphite Structure by Polarization Dependence of near-Edge X-Ray-Absorption Fine Structure. Phys. Rev. B 2000, 62, R6053-R6056.

(123) Frazer, B. H.; Gilbert, B.; Sonderegger, B. R.; De Stasio, G. The Probing Depth of Total Electron Yield in the Sub-keV Range: TEY-XAS and X-PEEM. Surf. Sci. 2003, 537, 161-167.

(124) Laurila, T.; Sainio, S.; Jiang, H.; Koskinen, J.; Koehne, J.; Meyyappan, M. The Role of Extra Carbon Source during the PreAnnealing Stage in the Growth of Carbon Nanofibers. Carbon 2016, $100,351-354$. 